\title{
Waterdrop Collisions With Solid Surfaces
}

\author{
Olive G. Engel
}

\begin{abstract}
An approach is described to the difficult problem of the dynamics of an impinging water sphere. The flow in the impact plane is traced chemically. The stages in the collapse of the water mass and of its radial flow are shown in photographs that were taken with a high-speed motion-picture camera. Empirical determinations of the time dependence of the impact force and of the radial flow velocity are reported. The possible occurrence of cavitation in the radial flow is considered. A semiempirical analysis based upon various simplifying assumptions leads to equations for the maximum impact pressure and for the rate of spread of the water after the collision.
\end{abstract}

\section{Introduction}

Erosion by waterdrop impact has recently received attention because of the damage that is done to highspeed aircraft on flying through rain. The problem is not new. Similar erosion is produced in steam turbines by the waterdrops in wet steam.

The destructive force causing this erosion results from the collision of the solid surface with the waterdrop. At high impact velocities a waterdrop acts as though it were a hard sphere. Unlike a sphere of hard material, it undergoes an outward radial flow of very high velocity as a result of the collision.

The question as to the order of magnitude of the pressure that is developed in collisions of waterdrops against solid surfaces has been discussed since the first studies of this erosion were begun approximately 30 years ago. Efforts have been made both to calculate the pressure from theoretical considerations and to measure it experimentally.

\section{Behavior of a Liquid Drop on Collision With a Flat Solid Surface}

The process of collision of one solid against another solid is well known in elasticity theory. The collision of small solids with a body of liquid has also been investigated in water-entry problems. The collision of liquid drops against a solid surface, however, has been studied very little. Some preliminary work for the purpose of obtaining a better understanding of waterdrop-to-solid collisions is described in this paper. This work includes (1) a chemical mapping of the radial water flow in the impact plane, (2) the use of high-speed motion-picture photography to "stop" the motion of the drop sufficiently during the collision so that the stages in the transition from vertical to radial flow can be observed, and (3) the use of schlieren photography to study details in the radial flow. The following discussion applies to the case of collision of a waterdrop with a flat solid surface.

\subsection{Map of the Radial Water Flow}

Worthington [1] ${ }^{1}$ with the aid of spark photography made a study of the forms assumed by drops of

${ }_{1}^{1}$ Figures in brackets indicate the literature references at the end of this paper. liquids (mainly milk and mercury) after a vertical fall from heights up to 11 in. onto a horizontal surface. A tendency toward formation of radial arms was found by Worthington to increase both with the height of fall and with the size of the drop.

The radial flow of a waterdrop was mapped chemically at the National Bureau of Standards. To accomplish this, a very small crystal of sodium dichromate held on the point of a needle was placed in the bottom surface of a waterdrop just before it fell from a flat-nosed pipet. The solution of this oxidizing agent is heavier than water and remained in the bottom of the drop. The drop was then allowed to fall onto a glass plate covered with a filter paper that was previously wet with acidified starch and potassium iodide solutions. A typical starch-iodide print of the radial flow from a waterdrop that fell approximately $1.5 \mathrm{ft}$ is shown in figure 1 . The water that struck first, and which contained the sodium dichromate, washed to the periphery of the flow, as can be seen by the stronger starch-iodide color there. The water that struck last essentially did not flow.

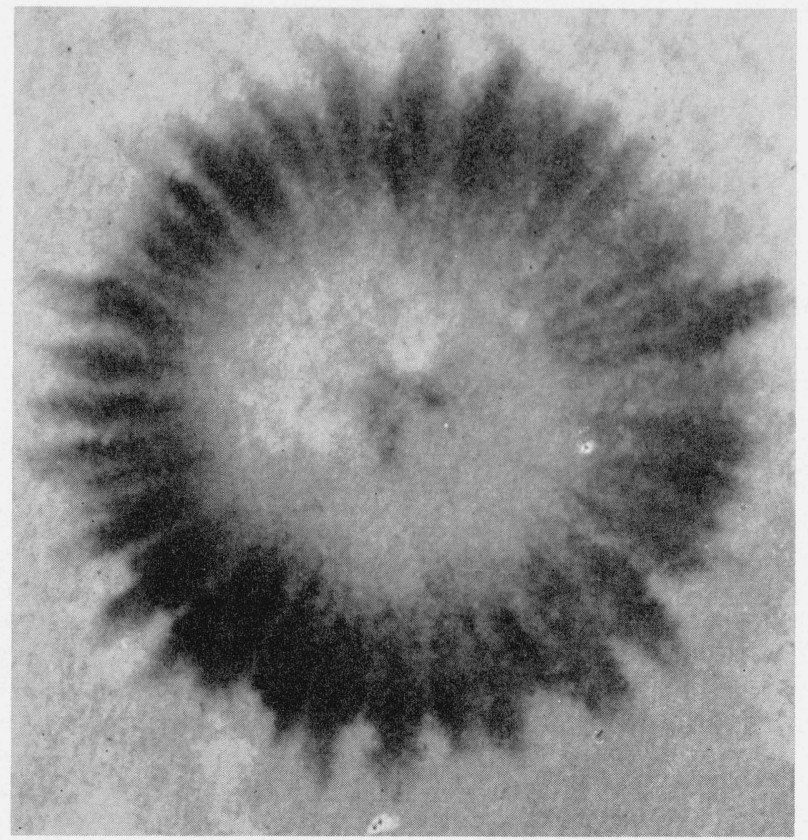

FIGURE 1. Starch-iodide trace of the radial flow of a waterdrop after collision with a solid surface. 
The radial arms in the structure show that the flow was channeled. It is conceivable that these channels are a result of the viscous drag between the solid surface and the flowing liquid, as was suggested by Worthington [2]. On the other hand, the observation of Worthington that the number of channels increases both with the size and with the height of fall of the drop suggests that the number of them may be related to the kinetic energy of the drop.

\subsection{Stages in the Collapse of a Waterdrop After Collision}

High-speed motion pictures can stop sufficiently the movement of a waterdrop after it has collided with a solid surface so that the horizontal flow can be observed. Figures 2, 3, 6, 7, and 9 are enlargements from high-speed moving pictures that were taken by 'T. C. Helimers, Jr., and HaroId Goodman, both of the Diamond Ordnance Fuze Laboratory, and by Warren P. Richardson of the NBS Technical Reports Section. The camera used is capable of taking 15,000 frames per second. The operating velocity at which any incident is photographed can be computed from the 60-cycle timer marks along the border of the film.

The steps in the collision and flow of a waterdrop after free fall through distances of about $1.5 \mathrm{ft}$ and of about $20 \mathrm{ft}$ are shown in figures 2 and 3, respectively. For the waterdrop collisions shown in these figures a very small crystal of sodium dichromate was placed in the bottom surface of the drop, and the drop was allowed to fall against a glass plate covered with a filter paper that was previously wet with acidified starch and potassium iodide solutions. A waterdrop oscillates between a vertical and a horizontal elongation as it falls. The collision shown in figure 2 occurred while the drop was elongated vertically; that shown in figure 3 occurred while the drop was elongated horizontally. It can be concluded from these pictures that the head of liquid of the drop, before it enters into radial flow, shows an ability to resist a change of shape during collision with a solid. This may be a result of its inertia, or of its viscosity, or surface tension.

\subsection{Schlieren Pictures of Impinging Waterdrops}

To accelerate waterdrops to their terminal velocity in air by free fall under the force of gravity, a tube was installed in a staircase well to extend through three stories of the Industrial Building of the National Bureau of Standards. This tube served the purpose of shielding the waterdrops from air currents so that they could be made to fall with little deviation on a predetermined spot on a glass plate located at the lower exit of the tube. A flat-nosed pipet, from which the drops originated, was mounted at the top of the tube.

If the solid surface against which the waterdrop is allowed to impinge is a glass plate, the stages in the radial flow of the drop can be photographed through the plate itself. A complete schlieren system was found to produce pictures from which the most infor-
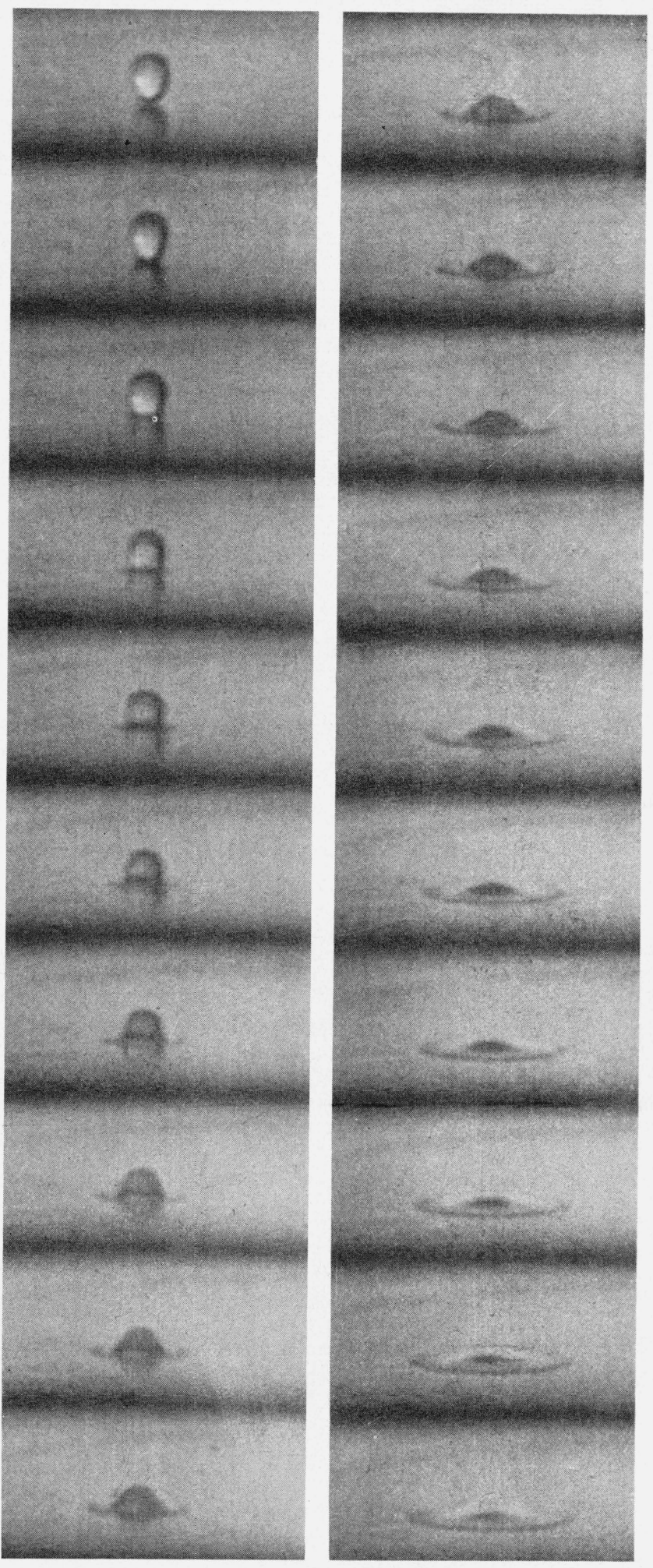

Figure 2. Stages in the flow of a waterdrop that fell from a height of approximately 1.5 feet. 

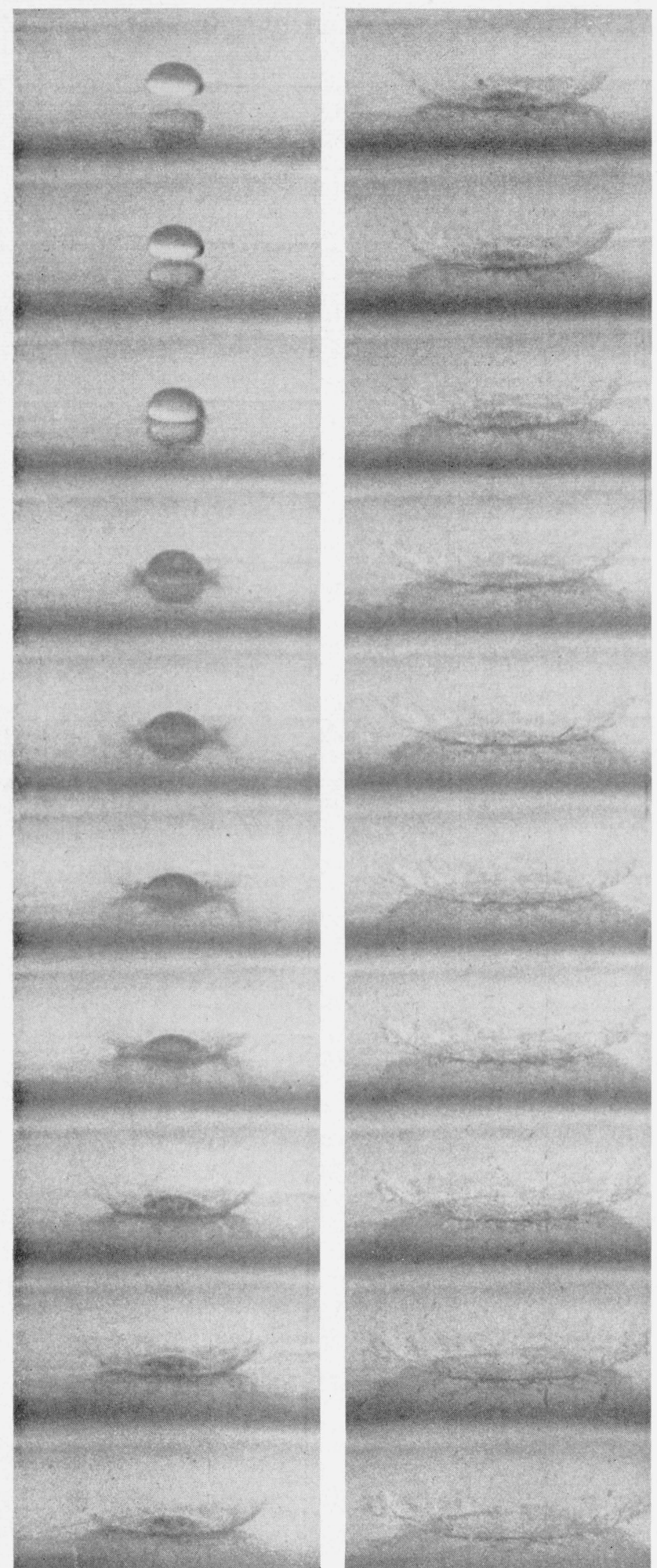

Figure 3. Stages in the flow of a waterdrop that fell from a height of approximately 20 feet.

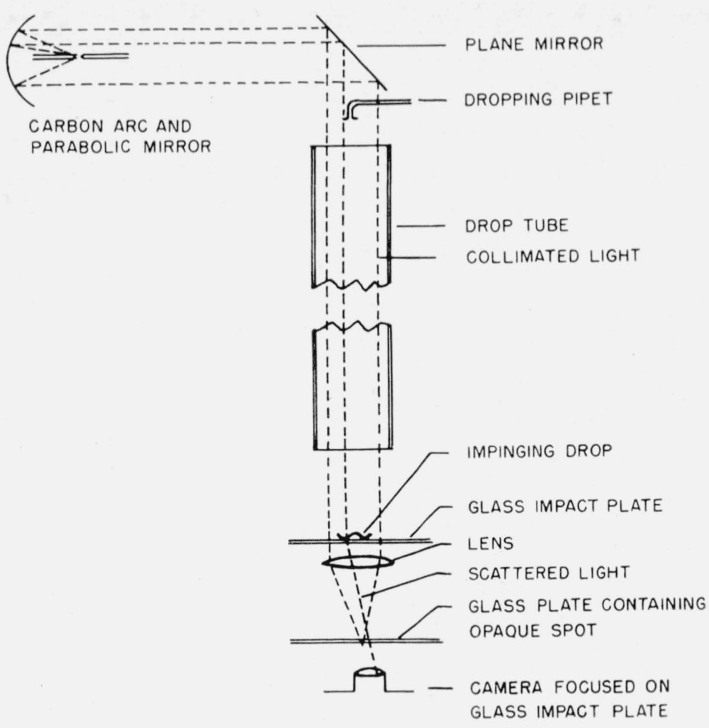

Figure 4. Elements of a schlieren system for photographing impinging waterdrops.

mation could be obtained. A sketch of the elements of the arrangement that was used is shown in figure 4. The arrangement was planned by Roland V. Shack of the NBS Optical Instruments Section.

\section{a. Schlieren Arrangement for Photographing an Impinging Waterdrop}

The light source was a carbon are searchlight that operated on a 40-amp current. The light was collimated by a parabolic mirror and was directed down the tube, through which the waterdrops fell, by means of a front-surface plane mirror. The tube was dyed black on the inside to reduce light scattering. The degree of collimation of the light was further improved by a circle of cardboard that was fastened to the exit of the tube and that allowed only the central core of the collimated beam to pass through to the glass plate.

The collimated light was transmitted through the glass plate and was focused by a lens. The sharp spot of light, which formed at the focal point of the lens, was received on an opaque barrier that had the same size and shape as the spot of light itself. This barrier was made by allowing the spot of light to fall on a spectroscopic plate that was rigidly mounted at the focal point of the lens in complete darkness. The opaque barrier served the purpose of a schlieren knife edge. The camera was mounted below the opaque barrier and was focused on the upper surface of the glass plate. Theoretically, only the light that was scattered by the impinging waterdrop would be deflected so as to enter the lens of the camera.

\section{b. Interpretation of the Schlieren Patterns}

To show how the light that was scattered by an impinging waterdrop would appear in pictures taken with the schlieren arrangement, four glass models of waterdrops were fashioned by L. Testa of the NBS Glassb lowing Shop. The models ranged 
from a solid glass sphere to an almost flattened glass disk. They are shown in figure 5, along with the corresponding photographs that were made when they were placed on the glass plate of the schlieren arrangement. The pictures numbered 1 through 4 in figure 5 are the glass models. The pictures having the corresponding primed numbers are the schlieren patterns that the models produced. A $50-\mathrm{mm}$ lens was used to photograph the schlieren patterns. The lens was kept at its widest aperture so that the depth of field was very short.

In picture 1 of figure 5 a hollow glass bead (black pointer) is shown with the solid glass bead. The schlieren patterns obtained from these beads show how difficult it is to tell the difference between a bubble in water and a protuberance from the water surface. The hollow bead produces only a somewhat larger high light in the schlieren pattern than the solid bead produces. Picture 2 of figure 5 shows a glass model of a waterdrop in the first stage of radial flow. The schlieren pattern produced by it is the same as that produced by the solid glass sphere but has a slightly larger diameter. A small difference in diameter is meaningful in this case because the geometry of the schlieren arrangement remained constant.

Pictures 3 and 4 of figure 5 show glass models of a waterdrop in more advanced stages of radial flow. The glass model in picture 4 has a ridge at the periphery of the radial flow, whereas that shown in picture 3 does not. Comparison of the structure of these models with their schlieren patterns shows that a flat area appears light in the schlieren pattern, regardless of whether it exists on a ridge or in a depression, whereas an area that has any degree of inclination to the reference plate on which the glass model is resting appears dark. Apparently the only way that the presence of a ridge or a depression can be identified is by the sloping area leading to it. The spherical residue in the center of the glass models in pictures 3 and 4 represents an inclined area and appears dark in pictures $3^{\prime}$ and $4^{\prime}$. In pictures $1^{\prime}$ and $2^{\prime}$ the schlieren patterns are also dark as is now expected because the glass models that produced them (pictures 1 and 2 ) have no area that does not have an inclination to the reference plane.

\section{c. Increase in Size of the Central High Light}

The schlieren patterns shown in pictures $1^{\prime}$ and $2^{\prime}$ of figure 5 have a central high light as do also those shown in pictures $3^{\prime}$ and $4^{\prime}$. This results because the glass sphere or spherical residue acts as a lens. If the spherical residue should become progressively more flattened, it should behave as a lens of longer focal length, and the high light should be seen to become larger and more diffuse. Although this effect is observed in schlieren pictures of the collision of real waterdrops with the glass plate, it is not observed in pictures $1^{\prime}$ through $4^{\prime}$ probably because the degree of curvature of the spherical residue in the glass models is not sufficiently different.

The first 10 frames of a collision incident of a real waterdrop impinging against a glass plate as photographed at a small oblique angle above the glass plate are shown in figure $6 . \mathrm{As}$ soon as the waterdrop contacts the glass and flows at all, it becomes a combination lens in which the lower lens has a small central flat area. Under this condition two foci exist, namely, that of the double-convex lens and that of the single planoconvex lens. As the solid surface moves further through the waterdrop the intensity at the focus for the double-convex lens decreases and that at the focus for the single planoconvex lens increases. When the head of water that
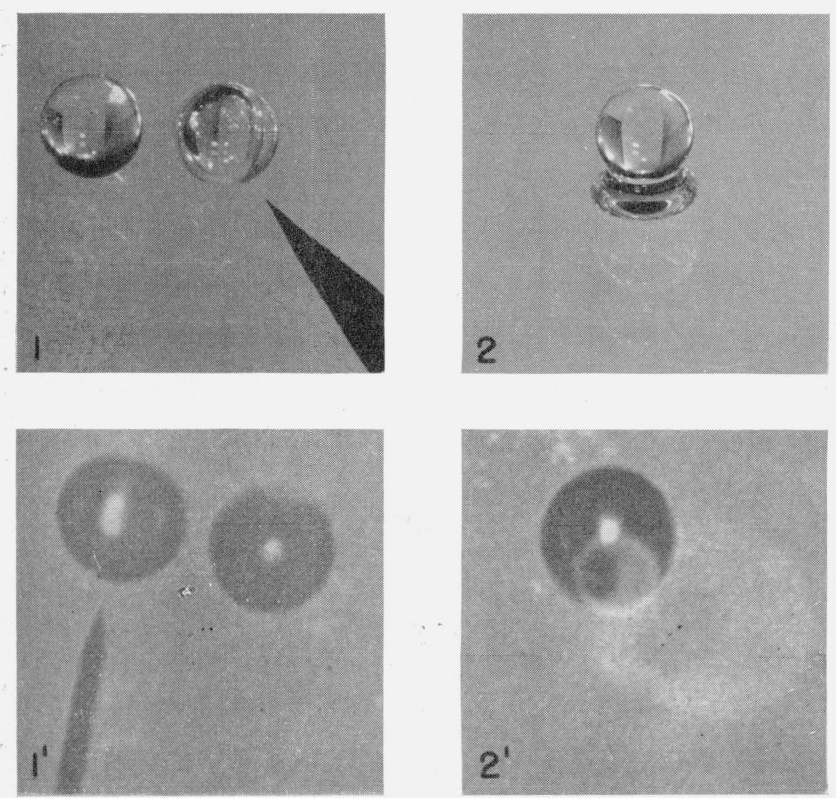
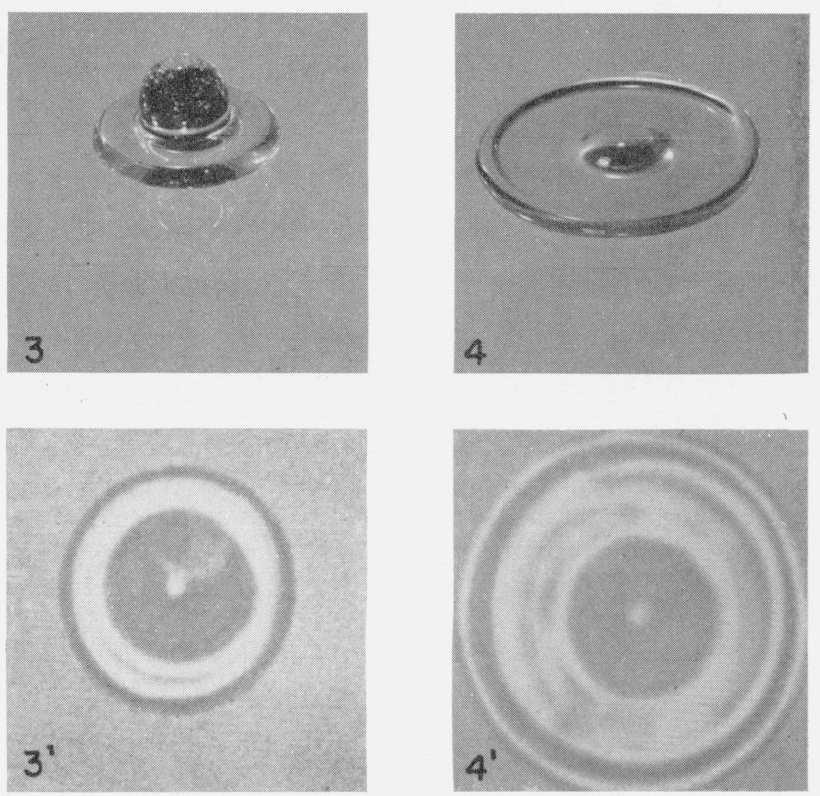

Figure 5. Glass models of waterdrops and their schlieren patterns (primed numbers). 
remains of the drop has decreased to a hemisphere, only the focus for the single planoconvex lens exists.

The focal length of a sphere of water measured from the point of contact with the flat surface on which it is resting, for rays making a small angle with the axis, is equal to its radius. The radius of curvature of the top of the drop in the first frame of figure 6 , as found by trial and error with dividers, was $0.36 \mathrm{~cm}$. The focal length of a single planoconvex lens of water measured from the plane side, which is in contact with the flat surface on which it is resting, for light that is incident on the curved surface of it, is 2.25 times its radius of curvature. As the radius of curvature of the water hemisphere that remains of the drop in the fifth frame of figure 6 was $0.51 \mathrm{~cm}$, its focal length at this stage of the collision was about
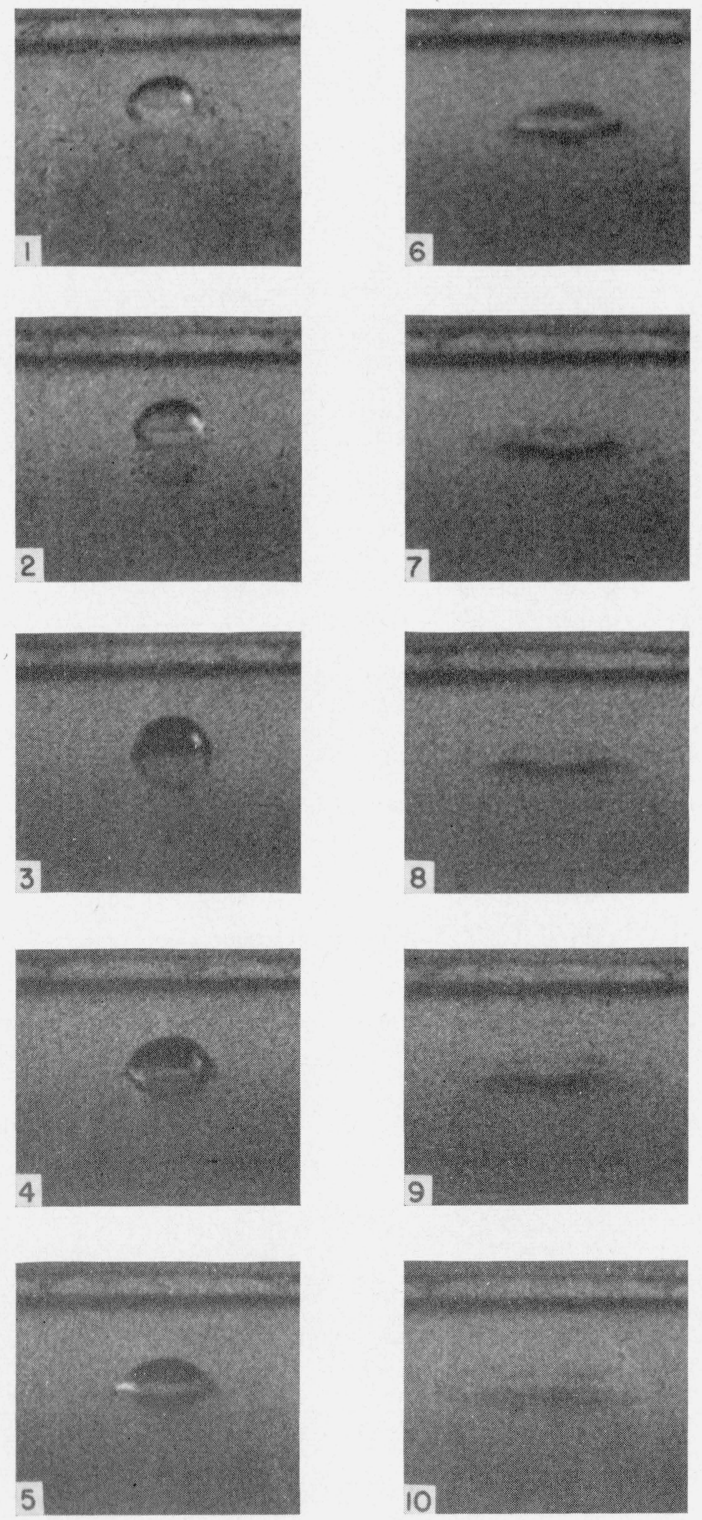

Figure 6. Stages in the collision of a waterdrop with a glass plate.
$1.15 \mathrm{~cm}$. The difference in the focal length of the water lens as it exists in frame 1 and in frame 5 is $0.79 \mathrm{~cm}$. As the depth of field of the camera used to take the schlieren photographs was less than $0.2 \mathrm{~cm}$, it seems reasonable that a perceptible increase in the size of the high light in the center of the schlieren patterns of a waterdrop should be observed during the course of a collision between the waterdrop and a glass plate. This effect can, indeed, be observed in frames 1 to 13 of figure 7 .

d. Lifetime of the Head of Water of a Drop That Collides at Its Terminal Velocity in Air With a Glass Plate

The glass models were placed directly in the center of the schlieren arrangement in line with the opaque barrier and with the center of the camera lens. The waterdrops were usually displaced from this position. This may explain why the schlieren patterns of the waterdrops show an additional high light on the side of the head of water of the drop as long as it exists as such. When the head of water just disappears so that only a disk of water in radial flow remains, the side high light and the central high light merge. For waterdrops impinging against a glass plate at their terminal velocity in air, the number of frames from the first point of impact to the merging of the two high lights indicates that the time required for the disappearance of the head of water of the drop is about 1 msec (millisecond).

The merging of the high lights seems to be accompanied by a flash of light, which unfortunately obscures the details in the radial flow for a space of 6 or 7 frames. The explanation of the light flash seems to be that the water lens at this stage of the flow of the drop acts in conjunction with the glass lens of the schlieren system to allow light to pass around the opaque barrier. The event occurs consistently.

\section{e. Structures in the Radial Flow}

On inspection of schlieren patterns produced by collision incidents between a glass plate and impinging drops, such as those shown in figure 7 , it is seen that the radial flow of the liquid appears dark. With reference to the observations made in regard to the schlieren patterns produced by the glass models, this appears to indicate that the radial flow of the liquid has some degree of inclination with respect to the glass plate. Worthington [1] made observations with drops of milk and mereury for low heights of fall that show that the liquid in radial flow is deepest at the periphery (see fig. 15).

In the schlieren pictures the periphery of the radial flow is bounded by a beady white line. The beady character of the periphery of flow can be detected as early as frame 5 in figure 7 . With reference to the schlieren patterns produced by the glass models, a white area is either the top of an elevation or the bottom of a depression. In the case of the white boundary of the radial flow it is most likely an elevated ridge. This conclusion seems to be supported in the pictures shown in figure 2 . 


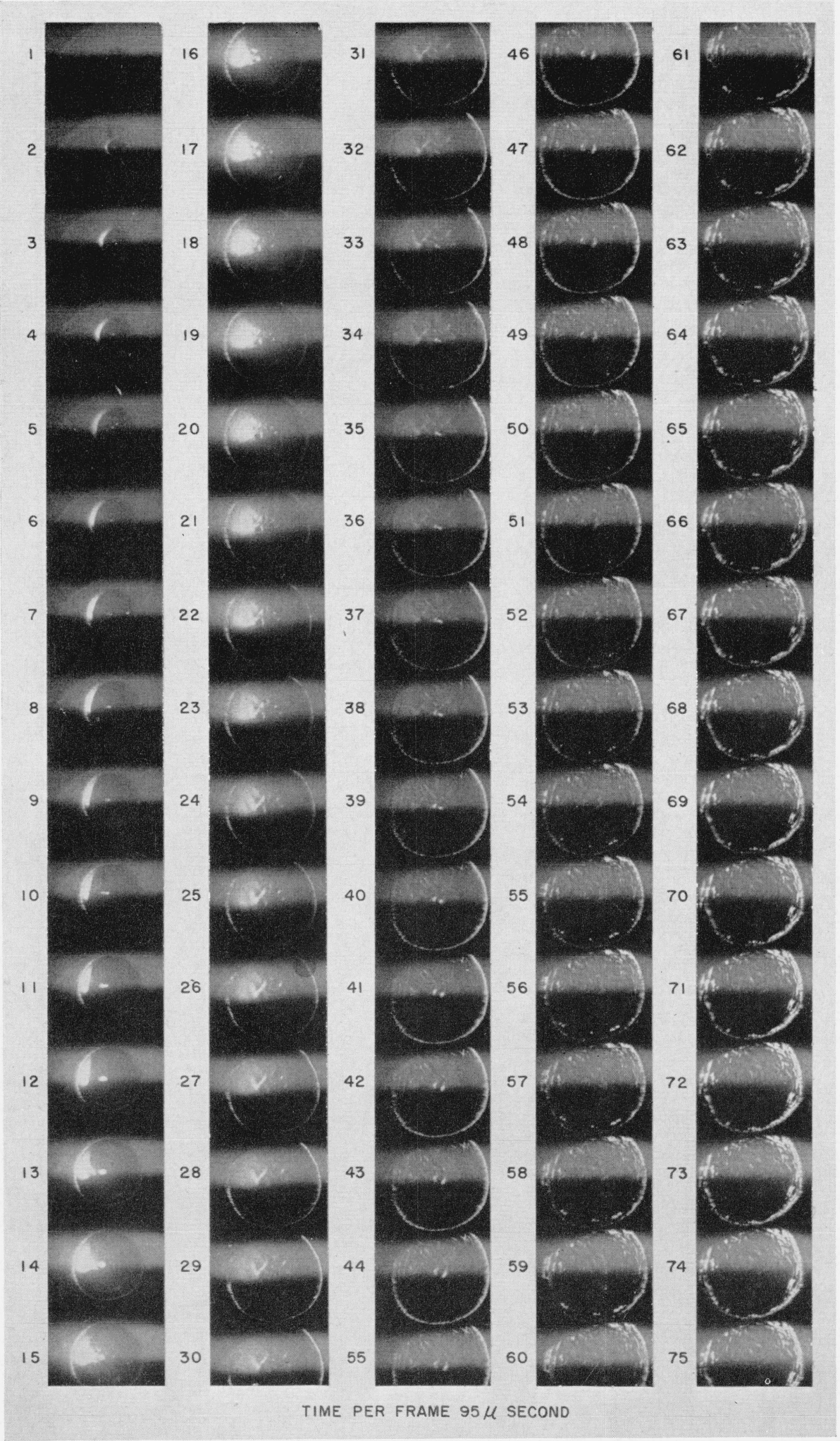

FIGURE 7. Schlieren patterns produced by the collision of a waterdrop with a glass plate 


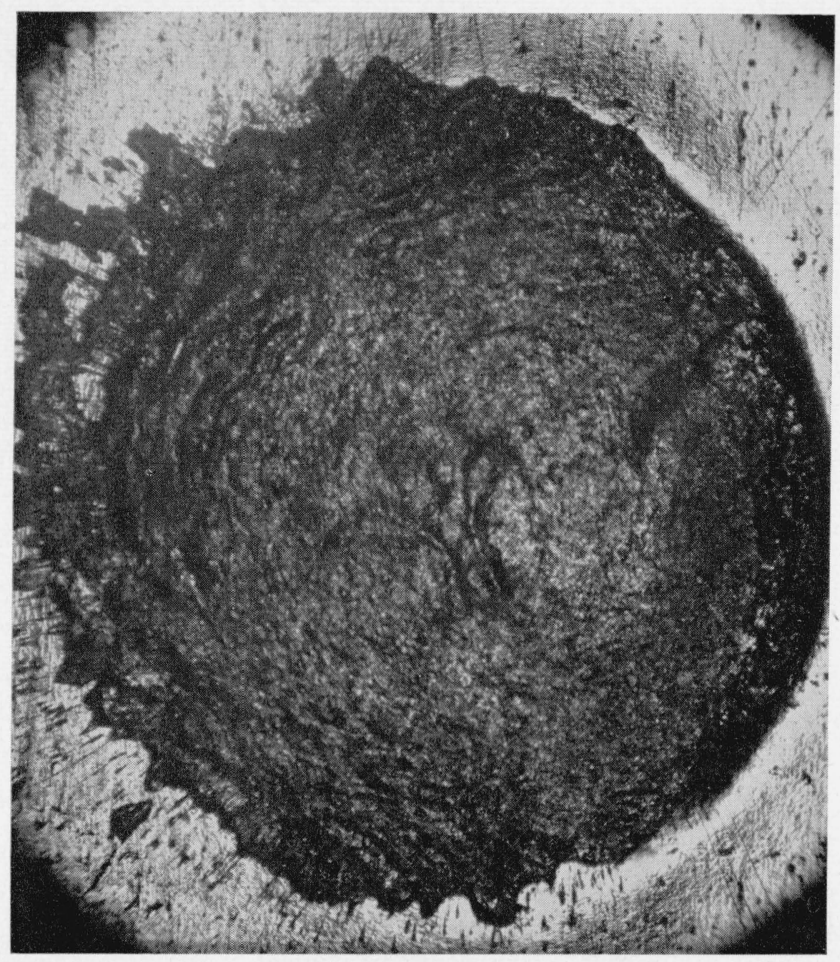

FiguRE 8. Ridges formed in the flow of a deforming lead pellet after collision.

Waves that run out over the radial flow can also be seen when high-speed pictures of a collision of a waterdrop with a glass plate are viewed with a projector. This effect is not visible in still enlargements.

Finally, inspection of pictures of various collision incidents between waterdrops and a glass plate shows that structures of some kind develop in the radial flow. The development of these structures follows the same general course. The central high light increases in size and finally takes on a definite structure. The structure grows in complexity and, after the central and side high lights of the drop merge, finally appears as separate white spots. That these white spots or areas are probably ridges and depressions can be seen from the flow that a deforming lead pellet undergoes after impact at very high speed against a metal plate. The flow of a deforming lead pellet is shown in figure 8 . In the case of the radial flow of water after impact, these ridges eventually vanish.

The explanation of why these ridges should form is not clear. The compressional wave started by the impact moves through the drop at the speed of sound in water and reflects from the opposite side of it as a tension wave. Thereafter it changes from tension to compression and back to tension alternately at the end of each round trip through the head of water that remains of the drop. It is possible that a standing wave may be produced as a consequence of the boundary conditions imposed on this alternate wave of compression and tension. On the other hand, it is possible that a solution that would predict standing waves may result from the continuity equation and the boundary conditions of the radial flow in the impact plane.

\section{f. Cavitation in the Radial Flow}

One of the most difficult aspects to understand in the problem of the erosion of durable structural materials as a result of high-speed waterdrop impingement is how initiation centers develop. It has been suggested by Albrecht Herzog of the Wright Air Development Center that incipient cavitation erosion may provide a first surface roughness from which erosion that results from waterdrop impingement could progress. A first surface roughening is observed both in the case of cavitation erosion and in the case of erosion by waterdrop impact. From this standpoint it is of interest to know whether or not any evidence of cavitation can be detected in the radial flow of a waterdrop after collision with a solid surface.

Cavitation is the formation of bubbles in a liquid. It occurs when the pressure on a liquid or in a small volume in a liquid drops below the value of the vapor pressure of the liquid at the temperature in question. The cavitation process itself is the spontaneous growth of gas nuclei (which already exist in the liquid) due to vaporization of the liquid across the liquid-vapor interface that each growing nucleus provides. When the pressure on the liquid is raised, or when the bubbles move out of a local low-pressure region in the liquid to a region of high pressure, the bubble-cavities collapse. It is the collapse of the cavities that produces the type of damage known as cavitation erosion.

There are at least two ways by means of which it is possible to explain theoretically how the conditions that produce cavitation may develop in a liquid drop after it collides with a solid surface at a velocity of sufficient magnitude. The first possible explanation is based on the very rapid radial flow of the liquid. It suggests that when the head of liquid of the drop has just disappeared into radial flow, the continued outward flow of the liquid under its own monemtum will produce a drop in pressure at the center of the spreading liquid disk. If the pressure at the center of the spreading liquid disk should fall below the vapor pressure of the liquid in question, cavitation may occur. This explanation has been advanced by Dr. Herzog. 'That a liquid drop may break in the center of the radial flow is shown in the work of Worthington [1]. He found that impinging drops of mercurv break in the center and form a ring rather than a solid disk of liquid.

The second possible explanation of how cavitation conditions may be realized is based on the alternating wave of compression and tension that exists in the head of liquid that has not yet become part of the radial flow of the drop. At the first instant of collision a wave of compression, which is initiated by the impact, moves through this head of liquid to the top of the drop. Here it reflects from the free liquid-toair surface as a tension or negative pressure wave. This negative pressure wave moves back through the 
drop to the impact surface. It must, in fact, be focused to a very small area of the impact surface by the curvature of the top of the drop in much the same way as ultrasonic waves in water may be focused by a watch glass. The returning negative pressure wave adds algebraically to the compressional wave that is still being initiated at the impact surface as a result of the collision. Since, however, the impact pressure has been decreasing steadily during the time interval in which the first compressional wave front made its round trip through the waterdrop and returned as a negative pressure wave, the net pressure is negative. The extent to which the net pressure is negative depends on the rate of decrease of the impact pressure with time. This explanation of how cavitation conditions may be produced in a liquid drop after collision with a solid surface is due to the late Francis E. Fox of the Catholic University.

Some slight evidence was found for what might be cavitation in schlieren pictures of a waterdrop collision with a glass plate. At the suggestion of Virginia Griffing of the Catholic University, waterdrops saturated with argon gas were used in an effort to show that the evidence that was observed was actually produced by bubbles in the water. Dir. Griffing has found that cavitation occurs readily in water that has been saturated with argon gas.

Enlarged pictures of 75 consecutive frames from one of the collisions of a drop of argon saturated water with the glass impact plate are shown in figure 9. In this figure the life cycle of a bubble can be followed from frame 19 to frame 33 . The appearances, which are described here, are more readily seen in the original photographs than in the halftone reproductions. The bubble appears slightly to the right of and below the center of the radial flow in frame 19. It grows in size and distinctness, then diminishes, and eventually vanishes in frame 33 . As the time per frame for this collision incident was 93 $\mu$ sec, the lifetime of this bubble was $1.4 \mathrm{msec}$. This is a reasonable lifetime for a cavitation bubble [3]. This bubble made its appearance shortly after the head of water of the drop vanished into radial flow in frame 14

A second bubble life cycle can also be followed in figure 9 . This bubble appears in frame 24 on a level with the bubble that has just been discussed but about $1 / 8$ in. from the periphery at the left side of the radial flow. It increases in brightness and then dims virtually to extinction in frame 34 . The lifetime is 0.9 msec. In frame 42 a white spot, which may be the same bubble, starts to increase in brightness. It is about $1 / 16 \mathrm{in}$. from the leit periphery of the radial flow. It continues to increase in brightness and, at frame 60 , that is, 1.7 msec aiter its reappearance, it driîts into the left periphery of the radial flow.

Two bubbles slightly below and to the leit of the center of the radial flow can be observed from frame 31 to frame 45 . In frame 46 the characteristic white semicircle which seems to be associated with a surface bubble (possibly due to the raised rim of water that accompanies such a bubble) appears. One of the bubbles has broken by irame 52 , as is seen by the train of ripples that forms from the white semicircle. The other bubble becomes obscure at about frame 59 or 60 . A bubble floating on a liquid suriace depresses the liquid level below it and is accompanied by a stable meniscus or raised rim of liquid at its periphery. If the bubble collapses, the differences in liquid level are no longer stable. This condition will produce waves. These waves are predominantly capillary waves since the wave length is small.

The collision incident for a drop of ordinary hydrant water against the glass impact plate is shown in figure 7. A small white spot can be seen very slightly to the right of the center of the radial flow in frame 14. This spot grows in brightness to about frame 25. It then becomes progressively dimmer until it disappears at about frame 40 . This is a lifetime of about 2.5 msec. The rise of a bubble to the suriace and its collapse in the suriace can also be traced in this figure. The first evidence of this bubble is in frame 29 at about the center of the radial flow. By frame 42 it has developed into two white semicircles enclosing a small white spot. As has already been noted, this structure seems to be typical of a surface bubble. In frame 46 the tiny central white spot is more diffuse and the enclosing semicircular white spots have started to split into semicircular lines. In the succeeding frames these lines spread out. In frame 54 they are almost concentric circles such as are formed when a pebble is dropped in a pool. Some vestige of this configuration can still be seen in frame 73 .

The fact that the bubble life cycles that have been pointed out seem to start after the head of water of the drop has vanished must not be construed as invalidating the second of the explanations of how cavitation conditions may be realized in a liquid drop after impact against a solid surface. This was called to my attention by Phillip Eisenberg of the Office of Naval Research. It may be necessary for the negative pressure wave to return to the impact surface many times before the bubble nuclei have sufficient time to grow to a size that can be seen.

In evaluating the evidence, which has just been presented, for the possible existence of cavitation in a waterdrop after collision with a solid surface, it is important that the effect of the impact velocity should be considered. The terminal velocity of a large waterdrop in air is [4] about $26.9 \mathrm{ft} / \mathrm{sec}(820$ $\mathrm{cm} / \mathrm{sec})$. One can hardly extrapolate the evidence for cavitation found at this velocity to what may be found at airplane flight velocities, for example, which are of the order of $900 \mathrm{ft} / \mathrm{sec}$. At the high impact velocities at which erosion is actually observed as a result of waterdrop impact the negative pressure would be very much increased and the possibility that cavitation may occur consequently much enhanced. This would follow from either of the explanations of how cavitation conditions may be produced as a result of the impact of a waterdrop against a solid surface. 


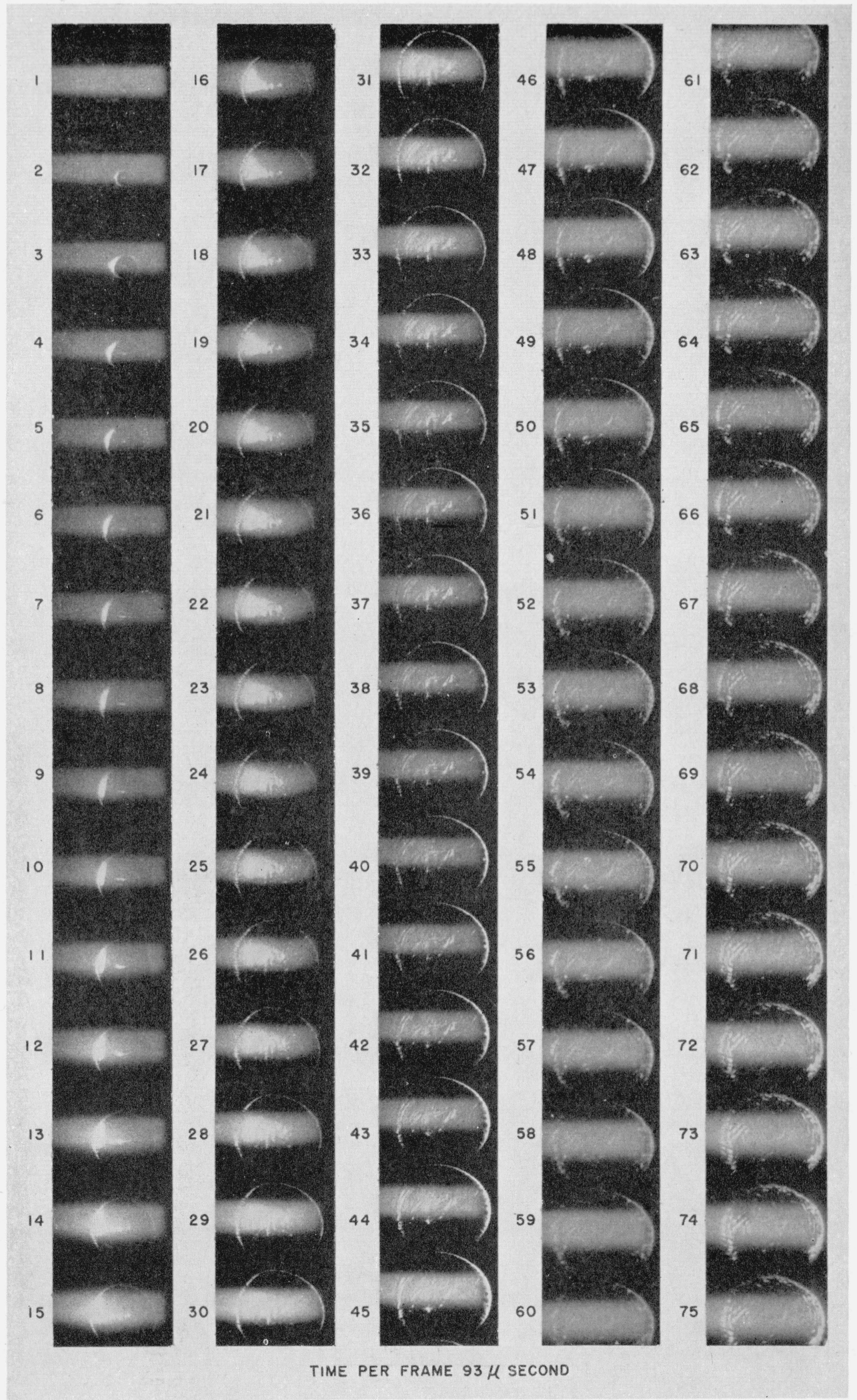

FIGURE 9. Schlieren patterns of the collision of a drop of argon saturated water with a glass plate. 


\subsection{Time Dependence of the Impact Force}

It is of interest to know something about the order of time during which the impact force developed by the impingement of a freely falling waterdrop against a solid surface is effective. A preliminary attempt to measure this has recently been made by Lawrence Fleming of the Diamond Ordnance Fuze Laboratory, using a thin disk of barium titanate ceramic.

The barium titanate disk that was used was about $1 \mathrm{~cm}$ in diameter. It was coated with silver on both sides to make it conducting and was fastened with polystrene adhesive to a small metal base. The complete unit was then coated with polystyrene lacquer to prevent the water of the drop from short circuiting the silver layers. Leads from the silver layers were connected to a Dumont 304 oscilloscope.

The barium titanate unit was placed on a glass plate that was located below the exit of the tube through which the waterdrops were allowed to fall. The waterdrops struck the barium titanate disk after a free fall of approximately $40 \mathrm{ft}$. The traces that appeared on the oscilloscope screen as a result of the waterdrop collisions against the barium titanate were photographed directly. One of these traces with the time base marker is shown in figure 10. The time between cycles in the time base marker is $100 \mu$ sec.

It can be seen from the oscilloscope trace that the impact force is applied very suddenly. The movement of the point of light on the oscilloscope screen due to the increasing force was too rapid to leave a
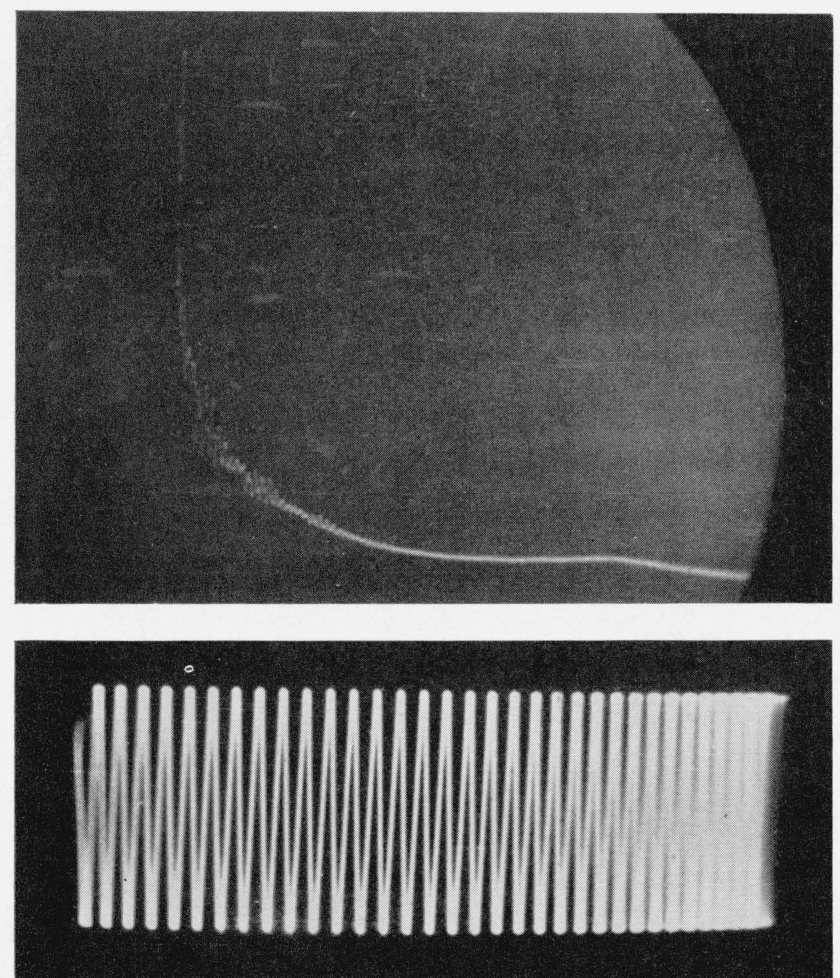

Figure 10. Oscilloscope trace of the decay of the force produced by a waterdrop collision. trace in the photograph. The decay curve only is visible. The force undergoes a rapid decay to zero in approximately the space of $1 \mathrm{msec}$. As has been noted in section $2.3, \mathrm{~d}$, this is also approximately the time during which the head of water of the drop just vanishes.

\subsection{Time Dependence of the Flow Velocity}

The experimental values of radius of flow at regular intervals of time for the spread of a drop of water after impact at its terminal velocity in air against a glass plate are shown graphically in figure 11. The data for this graph, which are given in table 1, were secured from 50 consecutive pictures of the history of the collision of a waterdrop against a glass plate. The pictures were the first 50 frames of figure 7. The measurements of the diameter of the radial flow were made under low magnification with dividers and a steel rule graduated to 0.01 in. Each experimental diameter is the average of two measurements. The values plotted on the graph have been multiplied by a magnifying factor of 2.7. The magnifying factor was determined from a series of measurements of the diameter of the maximum spread of drops that were obtained from the same pipet and that fell approximately the same distance. The maximum radius of flow found in this way was $2.3 \mathrm{~cm}$. The maximum radius of flow in the schlieren photographs occurred at frame 44 and was $0.85 \mathrm{~cm}$. The quotient of these values of the maximum spread radius produced the magnifying factor.

Ambiguity exists as to the exact time at which the flow was initiated. Any radius of flow that is less than the radius of the drop itself is obscured by the drop. Extrapolation of the curve indicates that flow began not at time equal to zero on the graph but at a time equal to approximately $152 \mu$ sec. That is, the origin of the curve is translated 152 $\mu$ sec along the abscissa because, of necessity, the radius of flow is zero at zero time, which is taken to be the first point of impact.

The slope of the curve of radius against time is the velocity of the radial flow. This curve is also shown in figure 11. The observed points are those obtained directly from the radius-against-time curve. The velocities are plotted in terms of the terminal

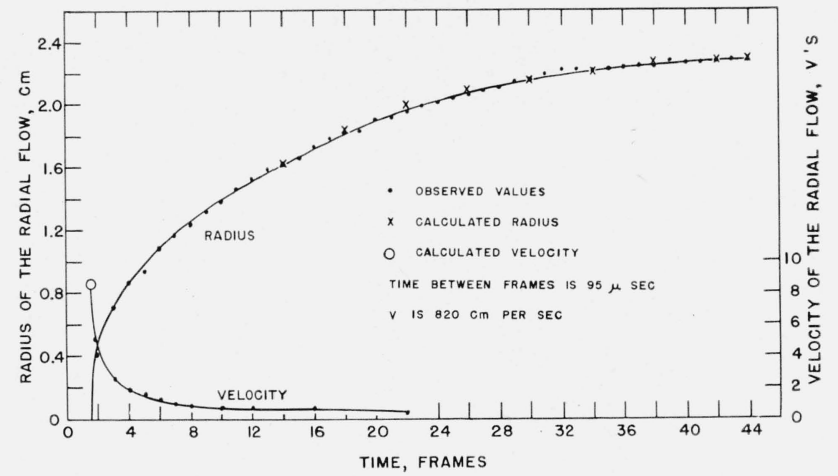

FIGURE 11. Time dependence of the radius of flow and of the flow velocity of an impinging waterdrop. 
TABLE 1. Values of the radius of flow for the waterdrop collision shown in figure 7

\begin{tabular}{|c|c|c|c|c|c|}
\hline Frame & Time & $\begin{array}{l}\text { Experi- } \\
\text { mental } \\
\text { measured } \\
\text { radius }\end{array}$ & $\begin{array}{l}\text { Magnify- } \\
\text { ing factor }\end{array}$ & $\begin{array}{l}\text { Experi- } \\
\text { mental } \\
\text { real radius }\end{array}$ & $\begin{array}{l}\text { Calcu- } \\
\text { lated real } \\
\text { radius }\end{array}$ \\
\hline $\begin{array}{l}1 \\
2 \\
3 \\
4 \\
5\end{array}$ & $\begin{array}{l}\text { sec } \\
.00003_{8} \\
.00013_{3} \\
.00022_{8} \\
.00032_{3}\end{array}$ & $\begin{array}{l}c m \\
0.15_{2} \\
.15_{2} \\
.26_{7} \\
.32_{3} \\
.35_{0}\end{array}$ & $\begin{array}{l}2.7 \\
2.7 \\
2.7 \\
2.7 \\
2.7\end{array}$ & $\begin{array}{l}c m \\
0.41_{2} \\
.41_{2} \\
.72_{0} \\
.87_{1} \\
.94_{6}\end{array}$ & \begin{tabular}{c}
$c m$ \\
\hdashline-1 \\
-1.
\end{tabular} \\
\hline $\begin{array}{r}6 \\
7 \\
8 \\
9 \\
10\end{array}$ & $\begin{array}{l}.00041_{8} \\
.00051_{3} \\
.00060_{8} \\
.00070_{3} \\
.00079_{8}\end{array}$ & $\begin{array}{l}0.40_{1} \\
.43_{2} \\
.45_{7} \\
.49_{2} \\
.51_{2}\end{array}$ & $\begin{array}{l}2.7 \\
2.7 \\
2.7 \\
2.7 \\
2.7\end{array}$ & $\begin{array}{l}1.0_{8} \\
1.1_{7} \\
1.2_{3} \\
1.3_{3} \\
1.3_{8}\end{array}$ & 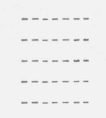 \\
\hline $\begin{array}{l}11 \\
12 \\
13 \\
14 \\
15\end{array}$ & $\begin{array}{l}.00089_{3} \\
.00098_{8} \\
.0010_{8} \\
.0011_{8} \\
.0012_{7}\end{array}$ & $\begin{array}{l}.54_{4} \\
.56_{6} \\
.589 \\
.59_{6} \\
.61_{7}\end{array}$ & $\begin{array}{l}2.7 \\
2.7 \\
2.7 \\
2.7 \\
2.7\end{array}$ & $\begin{array}{l}1.4_{7} \\
1.5_{3} \\
1.59 \\
1.6_{1} \\
1.67\end{array}$ & 1. $6_{1}$ \\
\hline $\begin{array}{l}16 \\
17 \\
18 \\
19 \\
20\end{array}$ & $\begin{array}{l}.0013_{7} \\
.0014_{6} \\
.0015_{6} \\
.0016_{5} \\
.0017_{5}\end{array}$ & $\begin{array}{l}.64_{4} \\
.66_{3} \\
.67_{3} \\
.67_{8} \\
.70_{7}\end{array}$ & $\begin{array}{l}2.7 \\
2.7 \\
2.7 \\
2.7 \\
2.7\end{array}$ & $\begin{array}{l}1.7_{4} \\
1.7_{9} \\
1.8_{2} \\
1.8_{3} \\
1.9_{1}\end{array}$ & $1.8_{4}$ \\
\hline $\begin{array}{l}21 \\
22 \\
23 \\
24 \\
25\end{array}$ & $\begin{array}{l}.0018_{4} \\
.0019_{4} \\
.0020_{3} \\
.0021_{3} \\
.0022_{2}\end{array}$ & $\begin{array}{l}.71_{1} \\
.72_{6} \\
.73_{9} \\
.74_{8} \\
.75_{7}\end{array}$ & $\begin{array}{l}2.7 \\
2.7 \\
2.7 \\
2.7 \\
2.7\end{array}$ & $\begin{array}{l}1.9_{2} \\
1.9_{6} \\
2.0_{0} \\
2.0_{2} \\
2.0_{4}\end{array}$ & 1.99 \\
\hline $\begin{array}{l}26 \\
27 \\
28 \\
29 \\
30\end{array}$ & $\begin{array}{l}.0023_{2} \\
.0024_{1} \\
.0025_{1} \\
.0026_{0} \\
.0027_{0}\end{array}$ & $\begin{array}{l}.76_{3} \\
.77_{7} \\
.78_{4} \\
.79_{5} \\
.80_{3}\end{array}$ & $\begin{array}{l}2.7 \\
2.7 \\
2.7 \\
2.7 \\
2.7\end{array}$ & $\begin{array}{l}\text { 2. } 0_{6} \\
2.1_{0} \\
2.1_{2} \\
2.1_{5} \\
2.1_{7}\end{array}$ & \begin{tabular}{c}
$2.1_{0}$ \\
\hdashline$-1.1_{7}$
\end{tabular} \\
\hline $\begin{array}{l}31 \\
32 \\
33 \\
34 \\
35\end{array}$ & $\begin{array}{l}.0027_{9} \\
.0028_{9} \\
.0029_{8} \\
.0030_{8} \\
.0031_{7}\end{array}$ & $\begin{array}{l}.81_{3} \\
.82_{6} \\
.82_{6} \\
.82_{0} \\
.82_{7}\end{array}$ & $\begin{array}{l}2.7 \\
2.7 \\
2.7 \\
2.7 \\
2.7\end{array}$ & $\begin{array}{l}2.1_{9} \\
2.2_{3} \\
2.2_{3} \\
2.2_{2} \\
2.2_{3}\end{array}$ & 2. $2_{2}$ \\
\hline $\begin{array}{l}36 \\
37 \\
38 \\
39 \\
40\end{array}$ & $\begin{array}{l}.0032_{7} \\
.0033_{6} \\
.0034_{6} \\
.0035_{5} \\
.0036_{5}\end{array}$ & $\begin{array}{l}.82_{8} \\
.83_{3} \\
.83_{6} \\
.84_{6} \\
.84_{1}\end{array}$ & $\begin{array}{l}2.7 \\
2.7 \\
2.7 \\
2.7 \\
2.7\end{array}$ & $\begin{array}{l}2.2_{4} \\
2.2_{5} \\
2.2_{6} \\
2.2_{8} \\
2.2_{7}\end{array}$ & $2.2_{6}$ \\
\hline $\begin{array}{l}41 \\
42 \\
43 \\
44\end{array}$ & $\begin{array}{l}.0037_{4} \\
.0038_{4} \\
.0039_{3} \\
.0040_{3}\end{array}$ & $\begin{array}{l}.83_{8} \\
.84_{6} \\
.84_{8} \\
.85_{1}\end{array}$ & $\begin{array}{l}2.7 \\
2.7 \\
2.7 \\
2.7\end{array}$ & $\begin{array}{l}\text { 2. } 2_{6} \\
2.2_{9} \\
2.29 \\
2.3_{0}\end{array}$ & $2.2_{9}$ \\
\hline
\end{tabular}

velocity, $V$, of the waterdrop, which was approximately $26.9 \mathrm{ft} / \mathrm{sec}(820 \mathrm{~cm} / \mathrm{sec})$. Expressing the radial flow velocity in terms of velocity $V$ that the drop had acquired at the instant at which it impinged against the glass plate makes it possible to see at a glance how much larger the radial flow velocity is than the impact velocity, for times just after the collision incident. It can also be seen at a glance that the radial flow velocity has dropped to the value of the impact velocity in less than $1 \mathrm{msec}$, that is, before the head of water that remains of the drop has disappeared into the radial flow. To express the values of the radial flow velocity in feet per second or in centimeters per second it is only necessary to multiply the values of the ordinate by 26.9 or by 820 , respectively.

The central and side high lights for this waterdrop merged, that is, the head of water of the drop vanished, at the end of 1.2 msec. Furthermore, the oscilloscope trace of force as a function of time shown in figure 10 for a waterdrop, that was obtained from the same pipet and that fell through the same dis- tance, indicates that the impact force vanished at the end of $1 \mathrm{msec}$. The velocity-against-time curve shown in figure 11 has the same shape as the oscilloscope trace of the force as a function of time shown in figure 10. This seems to indicate that the radial flow is propelled outward by the force of the impact. After the impact force vanishes the outward flow must continue, although at a much reduced velocity, under its own momentum.

\subsection{Collisions of Waterdrops Against Surfaces Hav- ing Different Degrees of Resilience or Smoothness}

That the flow characteristics of an impinging waterdrop depend strongly on the type of surface against which the collision occurs as well as on the velocity of the impact can be seen by comparing figures 2,3 , and 6 . For the study of this effect the high-speed camera was placed at a slight angle above the surface against which the waterdrop would impinge. The camera was placed rather far from the area on the surface where the waterdrop was expected to hit in order to increase the probability of getting a picture of the collision incident. Unfortunately, this resulted in a loss of detail so that enlargements of a few frames from the film in the region where the collision occurred proved to be of little value. The films were therefore studied in motion, using a motion-picture projector.

\section{a. Natural Rubber and GR-I Synthetic Rubber}

Impingement of a waterdrop on a sheet of natural rubber about 80 mils thick and on a sheet of GR-I synthetic rubber of the same approximate thickness resulted in flow configurations of the same general appearance. Quite a bit of spray in upward motion was produced in the first stage of the impact. This spray moved out with the periphery of the radial flow of the waterdrop. See, for example, the comparable configuration that resulted in the case of waterdrop impingement against wet filter paper shown in figure 3. The upward splash was more nearly vertical in the case of waterdrop impingement against natural rubber than in the case of waterdrop impingement against the synthetic rubber. Natural rubber is much more resilient than GR-I rubber. A steel sphere dropped on a sheet of natural rubber undergoes a considerable rebound. The same steel sphere dropped on a sheet of GR-I synthetic rubber hardly rises from the surface. It is possible that the difference in resilience of the two rubbers may explain the more nearly vertical spray in the case of the natural rubber. On the other hand, it is possible that the depression formed in the natural rubber as a result of the impact of the waterdrop was deeper and that the spray was directed up the more nearly vertical sides of the depression to produce the configuration of spray that was observed. The Shore " $A$ " hardness of the natural rubber was about 30 , whereas that of the GR-I synthetic rubber was about 20 . Although this static hardness measurement would seem to indicate that the greatest deformation should occur in the GR-I 
synthetic rubber, it cannot be taken as a certain indication of what to expect under dynamic conditions of loading.

\section{b. Dry Clean Glass}

The collision and radial flow of a waterdrop on a dry clean glass plate was smooth. There was a slight spray formation around the head of water of the drop as it was driven into radial flow (see fig. 6). The glass plates that were used for this study contained scratch blemishes.

\section{c. Iron Buffed and Iron as Received}

One side of an iron plate was buffed, the other side was retained in the as-received condition. Comparison of the flow configurations that resulted from the impingement of a waterdrop on each of these iron surfaces showed marked differences. In the case of the buffed surface there was essentially no upward splash of the water as a result of the collision. There was only a slight disturbance around the vanishing head of water of the drop with a very smooth outward radial flow of the water. Impingement of a waterdrop on the iron plate in the asreceived surface condition resulted in considerable upward splash of the water. The amount of spray that is formed as a result of a waterdrop-to-solid collision seems to be a function of the smoothness of the surface. The spray formation probably occurs at the time of maximum pressure.

\section{d. Fine Sand in a Petri Dish}

The collision of a waterdrop against fine moist sand in a petri dish appeared like an underground explosion of dynamite in diminutive. A crown of upward moving water splash and sand grains was produced by the impact. The print made in the sand by the collision consisted of a circular trench around a more or less undisturbed mound of sand in the center. The central mound of sand probably marked the stagnation point of the water flow from the drop. The circular trench was dug by the radial flow of the water. The configuration is quite interesting from the standpoint of how impinging waterdrops may dig pits in soft materials.

\section{Equation for the Impact Pressure Result- ing From Waterdrop-to-Solid Collisions}

An early estimate of the mean pressure that results from the collision of a solid surface with a water sphere was made by Honegger [5]. In his treatment the velocity of the center of gravity is the impact velocity, $V$, when the time, $t$, is zero (at the first point of impact). The velocity of the center of gravity is $V / 2$ at a time $\Delta t$ later. $\mathrm{He}$ assumed that the center of gravity during the time $\Delta t$ was displaced by the distance $d / 4$, where $d$ is the drop diameter. He then applied the impulse momentum equation in the form ${ }^{2}$

$$
M V / 2=P f \Delta t,
$$

where $M$ is the mass of the drop, $P$ is the pressure,

${ }^{2}$ The notation of other authors has been transcribed into the notation used in this paper. and $f$ is the mean contact area between the drop and the surface. He obtained in this way the equation for the pressure

$$
P=4 \times 10^{--6} V^{2},
$$

where $P$ is given in kilograms per square centimeter, if $V$ is expressed in centimeters per second.

De Haller [6] has written as an estimate of the im. pact pressure when a solid surface strikes a cylinder of water from the side the equation for the collision of two flat elastic bars. This is

$$
P=\frac{V \rho_{1} c_{1}}{1+\left(\rho_{1} c_{1} / \rho_{2} c_{2}\right)},
$$

where $\rho_{1}$ and $\rho_{2}$ are the density of the flat water bar and of the solid bar, respectively; $c_{1}$ and $c_{2}$ are the speed of sound in water and in the material of the solid bar, respectively; and $V$ is the relative speed of the water bar with respect to the bar of solid material. This equation neglects the curvature of the water cylinder.

From the results of the experimental work, which have been discussed in section 2 , it seems reasonable to think that a treatment similar to that of the elastic impact of solids may provide a reliable estimate of the impact pressure. However, the spherical shape of the waterdrop, which imposes the condition that compressional waves initiated in it by the collision are not all started simultaneously, must be taken into account.

Let the following assumptions be made:

(A) The first effect of the collision is the initiation of a compressional wave that starts to spread through the water sphere (see fig. 12). The water molecules of the spherical drop in the region traversed by the compressional wave attain a velocity in the direction in which the impinging solid surface is moving. This region of the drop consequently becomes slightly flattened. The velocity taken on by the water molecules in this region of the drop is dependent on the velocity of the impinging solid surface, but it is less than this velocity. Only a thin layer of water in direct contact with the impinging solid surface takes on a velocity that is identical with that of the solid

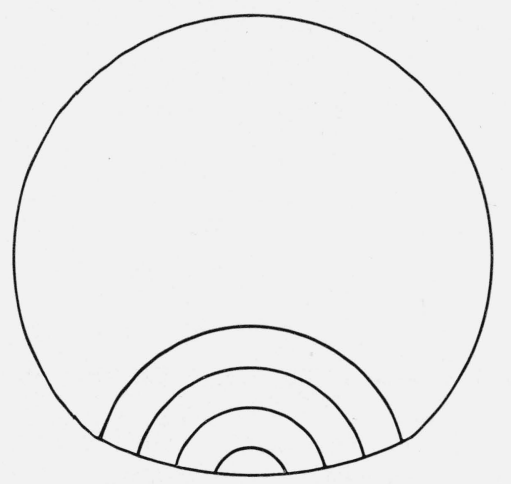

FIGURE 12. Schematic drawing indicating that the water sphere is flattened as a result of compressional wavelets initiated in it. 
surface. There is, furthermore, a velocity distribution among the water molecules in this region of the waterdrop. The following treatment is restricted to a consideration of the average velocity given to the water molecules in this region. Only the velocity in the direction in which the impinging solid surface is moving is considered.

(B) Maximum pressure, which is assumed to be reached at the end of the time interval $\Delta t$, is defined by the condition that the radiating compressional wavelet initiated at a point in the first instant of impact should just reinforce the compressional wavelet that is started at time $\Delta t$ later in producing momentum in the water sphere (see fig. 13). That is, the rate of change of momentum of the water molecules in the region where this reinforcement occurs is greater than that of any other of the water molecules of the drop because it is produced by the combined effect of the wavelets.

(C) The second effect of the collision is that the first liquid of the drop that encounters the solid surface during the time interval $\Delta t$ is displaced to form a thin cylinder under the drop (see fig. 14). The water of the shaded cap of the flattened drop of figure 14 is displaced into the ring the triangular cross section of which is bounded by the solid surface, the vertical boundary of the cylinder of water, and the curved boundary of the flattened waterdrop. The thin cylinder of water that forms and the region of the drop that is traversed by the compressional wave during the time interval $\Delta t$ are stippled in figure 14 . The thin cylinder of water is assumed to be in radial flow. The upper surface of it is termed the $\lambda$-plane. The head of water that remains of the drop (and which is separated from the surface of the impinging solid by the radially flowing thin cylinder of water) retains its shape except for a slight flattening (see figs. 2 and 3 ).

(D) As the collision continues, compressional wavelets are initiated at points of contact of the remaining water sphere with the $\lambda$-plane. In this

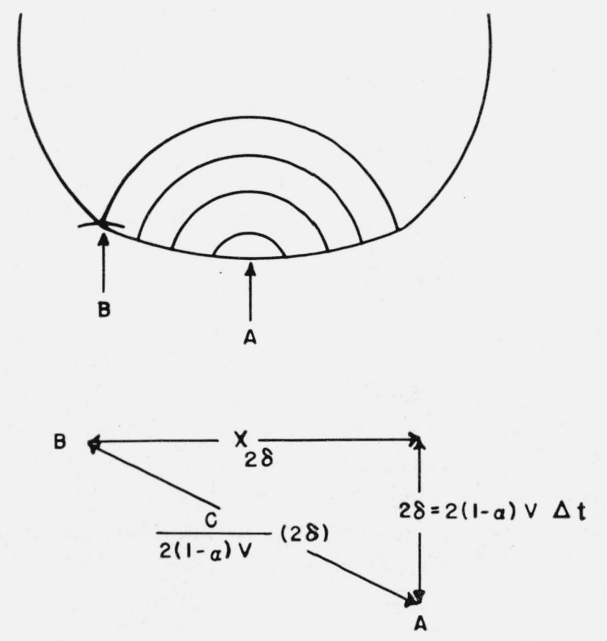

Figure 13. Schematic drawing that illustrates the assumption made in regard to the maximum pressure. sense the $\lambda$-plane, or upper boundary of the radial flow, may be considered as an effective striking surface.

Consider that the flat surface of a massive solid, moving with velocity $V$ in the z-direction, is just at the point of collision with a stationary water sphere. If the first point of contact between the water sphere and the flat solid surface is made the origin of a rectangular coordinate system, the equation of the sphere at the origin of the coordinates is

$$
x^{2}+y^{2}+\left(z-r^{\prime}\right)^{2}=r^{\prime 2}
$$

where the $x$ - and $y$-coordinates are in the plane of the colliding surface, and $r^{\prime}$ is the radius of the water sphere before the collision. For all points for which $y=0$, eq (4) reduces to the circle

$$
x^{2}+\left(z-r^{\prime}\right)^{2}=r^{\prime 2}
$$

in the $x z$-plane. The first point of collision between the solid surface and the water sphere is given by the equation for the circle of contact during the collision

$$
x^{2}+y^{2}=\left[R^{\prime}(z)\right]^{2},
$$

with $R^{\prime}(z)$ equal to zero.

Consider that the first result of the collision is the initiation of a compressional wave that immediately begins to spread through the water sphere (see fig. 12 and assumption $\mathrm{A}$ ). The water through which the compressional wave passes is given a velocity in the $z$-direction in which the surface of the massive solid was moving. As a result of this, the region of the water sphere that has been traversed by the compressional wave is slightly flattened in this direction. The radius of this flattened portion of the water sphere is $r$, and $r$ is greater than $r^{\prime}$.

The average velocity of the water in the region of the water sphere that has been traversed by the compressional wave may be written as $\alpha V$, where $\alpha$

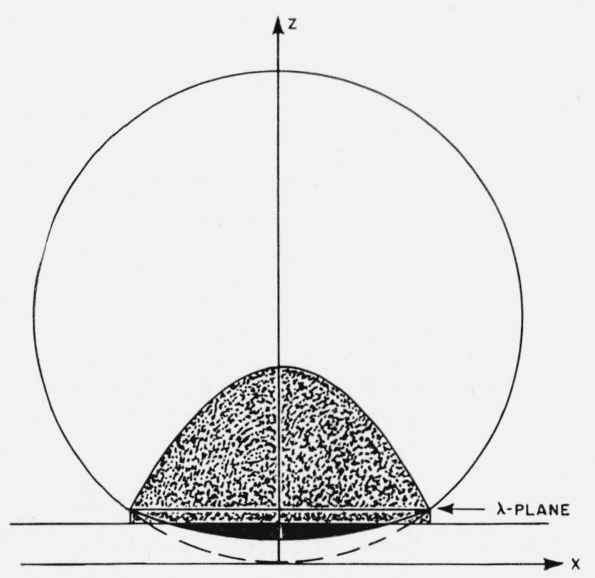

Figure 14. Cross section of the water sphere that shows schematically the locus of the vertical components of the compressional wave and the formation of the thin cylinder of water that is in radial flow. 
is a coefficient that tells what fraction of velocity $V$ is imparted to the water molecules on the average. As there is no marked attenuation of the amplitude of a compressional wave in water, the coefficient $\alpha$ must be governed mainly by the extent of divergence of the compressional wave as it spreads through the spherical drop. As the impact velocity increases for a series of waterdrop-to-solid collisions, the extent of flattening in the region of the drop that has been traversed by the compressional wave increases, the amount of divergence of the wave decreases, and the value of the coefficient $\alpha$ approaches unity.

Consider that the second result of the collision in the time interval $\Delta t$, during which the pressure reaches a maximum, is the displacement of the first water of the sphere that encountered the solid surface into a very thin cylinder of water that is in radial flow (see fig. 14 and assumption $\mathrm{C}$ ). The average velocity at which this displacement occurs is $(1-\alpha) V$. It can readily be shown that in the limit the volume of a very thin slice cut off a sphere is one-half the volume of a cylinder that has the same height and base area. Consequently, in the time $\Delta t$ in which the solid surface moving at the displacement velocity $(1-\alpha) V$ has moved a distance $\delta$ through the water sphere, where $\delta$ is the thickness of the cap of the flattened portion of the water sphere that is shaded in figure 14 , the $\lambda$-plane has moved a distance $2 \delta$. If the displacement velocity is constant, then the velocity $\bar{v}$ of the $\lambda$-plane is given by

$$
\bar{v}=2(1-\alpha) V .
$$

The massive striking surface is not at all slowed down as a result of the collision. It continues to move at the velocity $V$ in the fixed coordinate system. In the fixed coordinate system, therefore, it has traveled the distance $\delta^{\prime}$, where

$$
\delta^{\prime}=V \Delta t
$$

through space in the time interval $\Delta t$. It has, however, only displaced the water of the drop which has been traversed by the compressional wave (and which is moving in the same direction that it is moving) the distance

$$
\text { Hence } \quad \begin{aligned}
& \delta=(1-\alpha) V \Delta t . \\
& \delta=(1-\alpha) \delta^{\prime} .
\end{aligned}
$$

It can, furthermore, be shown that the radius, $r$, of the flattened portion of the water sphere, through which the compressional wave has passed and in which the water has the average velocity $\alpha V$ in the $z$-direction, is given by

$$
r=r^{\prime} /(1-\alpha) \text {. }
$$

After the short time $\Delta t$ the solid surface has moved a distance $\delta$ through that part of the water sphere that has been traversed by the compressional wave, the $\lambda$-plane has moved a distance $2 \delta$, and the compressional wavelet that was started at the impact instant at point $\mathbf{A}$ of figure 13 has moved $c / \bar{v}$ times a far as the $\lambda$-plane has moved, that is, $2 \delta c / \bar{v}$, and has reached point $B$. Here $c$ is the speed of the compressional wave in water. The radius of the circle of contact that is given by eq (6) with $R^{\prime}(z)$ replaced by $R(z)$ is now $x_{2 \delta}$. This, on assumption $\mathrm{B}$, is the point of maximum pressure because at this instant water molecules are being accelerated both by their own impact in the collision direction $z$ and by the compressional wavelet that was started at the impact instant and that has just arrived in their locality.

It is possible to solve for $\delta$, the thickness both of the shaded cap of water in figure 14 and of the disk of water in radial flow in the impact plane after the time $\Delta t$, in terms of the radius, $r$, of the flattened portion of the water sphere, the velocity of the striking surface, $V$, and the speed of the compressional wave, $c$. That is, using the right triangle shown in figure 13 ,

$$
x_{2 \delta}^{2}=\frac{4 c^{2} \delta^{2}}{\bar{v}^{2}}-4 \delta^{2}
$$

and from eq (5) with $r^{\prime}$ replaced by $r$,

$$
x^{2}=r^{2}-(z-r)^{2}=2 r z-z^{2},
$$

so that

$$
x^{2}{ }_{2 \delta}=4 r \delta-4 \delta^{2} \text {. }
$$

By subtracting eq (12) from eq (14), it is seen that

$$
\delta=r\left[\frac{\bar{v}}{c}\right]^{2} .
$$

According to assumption $\mathrm{B}$, maximum pressure occurs after time $\Delta t$. It is noteworthy that this maximum of pressure has been defined by an arbitrary assumption and is not to be regarded as necessarily coincident with the maximum of total force in figure 10. Actually, since the time $\Delta t$ as evaluated in section 4 for a collision of a waterdrop at its terminal velocity in air is $0.04 \mu \mathrm{sec}$, and since the time between cycles of the time base marker in figure 10 is $100 \mu \mathrm{sec}$, it is conceivable that it may exist at the left of this maximum. Furthermore, the maximum pressure as it is evaluated at the time $\Delta t$ in the following treatment is not an instantaneous maximum pressure. To obtain it the total mass of water set in motion during the time interval $\Delta t$, and consequently all the force that has acted to produce water momentum over the time interval $\Delta t$, is considered. The instantaneous force that is acting at the last instant of this time interval is not considered.

The average rate of change of momentum of water in that portion of the drop that has been traversed by the compressional wave over the time interval $\Delta t$ is $m \alpha V / \Delta t$, where $m$ is the total mass of water in the drop that is set into motion as a result of the collision. If all of the force that has acted to produce water momentum over the time interval $\Delta t$, at the end of which the radius of the circle of contact between the drop and the surface is $x_{2 \delta}$, is written as $\pi x_{2 \delta}^{2} P$, then

$$
P=m \alpha V /\left[\pi x_{2 \delta}^{2} \Delta t\right] .
$$


It is necessary to evaluate the mass of water, $m$, that was set in motion in the z-direction as a result of the collision. After the time $\Delta t$, part of this total mass of water, the mass $m^{\prime}$, is moving in horizontal flow in the impact plane as a result of the impact pressure. The mass moving horizontally is given by

$$
m^{\prime}=\frac{1}{2} x_{2 \delta}^{2} \pi \rho(2 \delta)=x_{2 \delta}^{2} \pi \rho \delta
$$

because in the limit, as has already been noted, a slice of thickness $2 \delta$ and radius $x_{2 \delta}$ cut off a sphere is just one-half the volume of a cylinder of the same height and base radius. Although the mass of water, $m^{\prime}$, is moving horizontally after the time $\Delta t$, it was given a velocity in the collision direction $z$ during this time interval because it was traversed by the compressional wave.

There is also the mass of water $m^{\prime \prime}$ in the head of water remaining of the drop that has a velocity in the $z$-direction because it has been traversed by the compressional wave. After the time $\Delta t$ the $\lambda$-plane has moved distance $2 \delta$, and the compressional wave has moved to the curved boundary of the stippled area of figure 14. It may be assumed that this volume of water has received the signal that collision has occurred. The lower boundary of this volume of water is the plane $z=2 \delta$, where the flattened sphere is at the origin of the coordinates. From eq (5) the z-coordinate of the points on the circle of radius $r$ from which wavelets of compression originated is

$$
z=r-\left(r^{2}-x^{2}\right)^{1 / 2} .
$$

$\Delta z$ is the distance the $\lambda$-plane moved up since the time that a compressional wavelet was started at the corresponding value of $x$. Then

$$
\Delta z=2 \delta-r+\left(r^{2}-x^{2}\right)^{1 / 2} .
$$

Let $z^{\prime}$ be the distance traveled above the plane $z=2 \delta$ by the compressional wavelet. Then

$$
z^{\prime}=\left[\frac{c}{2(1-\alpha) V}-1\right] \Delta z=\left[\frac{c}{\bar{v}}-1\right] \Delta z .
$$

The locus of the points $z^{\prime}$ gives the envelope of the components of the compressional wavelets in the collision direction $z$. Substituting the value of $\Delta z$ from eq (19) into eq (20),

$$
z^{\prime}=\left[\frac{c}{\bar{v}}-1\right]\left[2 \delta-r+\left(r^{2}-x^{2}\right)^{1 / 2}\right] .
$$

Rearranging,

\section{Letting}

$$
\left[\frac{\bar{v}}{c-\bar{v}}\right] z^{\prime}-2 \delta+r=\left(r^{2}-x^{2}\right)^{1 / 2} .
$$

$$
\frac{\bar{v}}{c-\bar{v}}=1 / \beta
$$

and squaring both sides of the equation

$$
x^{2}=-\left[\frac{z^{\prime}}{\beta}\right]^{2}+\frac{4 \delta z^{\prime}}{\beta}-\frac{2 r z^{\prime}}{\beta}-4 \delta^{2}+4 \delta r .
$$

Equation (24) is an expression for $x^{2}$ in terms of $z^{\prime}$; the enclosed volume of water, on substituting this value of $x^{2}$, is

$$
\pi \int_{z^{\prime}=0}^{z^{\prime}=\beta(2 \delta)} x^{2} d z^{\prime}=\beta \pi\left[-\frac{8}{3} \delta^{3}+4 \delta^{2} r\right] .
$$

The limit of the ratio of this volume over the volume of a cylinder of the same height and base area is also found to be one-half. Consequently, the mass of water $m^{\prime \prime}$ having the average velocity $\alpha V$ in the collision direction $z$ after the time $\Delta t$ is

$$
m^{\prime \prime}=\frac{\rho \pi \beta}{2} x_{2 \delta}^{2}(2 \delta)=\rho \pi \beta x_{2 \delta}^{2} \delta,
$$

and the total mass of water that was set into motion in the z-direction through having been traversed by the compressional wave is from eq (17) and (26)

$$
m=m^{\prime}+m^{\prime \prime}=(1+\beta) x_{2 \delta}^{2} \pi \rho \delta .
$$

The pressure, $P$, is then from eq (16)

$$
P=\frac{\left[(1+\beta) x_{2 \delta}^{2} \pi \rho \delta\right] \alpha V}{x_{2 \delta}^{2} \pi \Delta t} .
$$

Since

and since

$$
2 \delta=2(1-\alpha) V \Delta t=\bar{v} \Delta t
$$

$$
\beta=\left[\frac{c-\bar{v}}{\bar{v}}\right]
$$

so that $(1+\beta)=c / \bar{v}$,

$$
P=\frac{\alpha}{2}[c \rho V] .
$$

This is the well-known water-hammer equation multiplied by the factor $\alpha / 2$, which comes from the spherical shape of the waterdrop. It is noteworthy that the result that the pressure is some multiple of the water hammer pressure because of the spherical shape of the waterdrop could have been written down at once. There would, however, be no rational basis for guessing at the value of the multiple. The value of the coefficient $\alpha$ can be found empirically for waterdrop-to-solid collisions that can be stopped by high-speed motion pictures as is shown in section 4 . For collisions at very high impact velocities, the coefficient $\alpha$ is probably close to unity. Consequently, for these collisions a reliable estimate of the impact pressure can also be made from eq (30).

Equation (30) has been verified indirectly by de Haller [6], who has measured the impact pressure between a flat surface and a jet of water struck from the side, using a piezoelectric pressure gage having a piston diameter of $1.5 \mathrm{~mm}$. With his experi- 
mental arrangement he was really measuring the pressure under short water cylinders struck from the side. Since the side of a cylinder contains both the element of circle of a sphere and the element of straight line of a plane, the pressure under such water shapes should be greater than that given by eq (30) but less than that given by eq (3), which is the equation de Haller was using for the impact pressure. With his piezoelectric pressure gage de Haller found the highest pressure value for a velocity of $35 \mathrm{~m} / \mathrm{sec}$ to be $310 \mathrm{~kg} / \mathrm{cm}^{2}$. For this velocity eq (3) would predict a pressure of 490 $\mathrm{kg} / \mathrm{cm}^{2}$ if one assumes that the denominator on the right-hand side is essentially unity, that the speed of sound in water is $5,000 \mathrm{ft} / \mathrm{sec}$, and that $V$ is $\alpha V$, where $\alpha$ is about 0.9. Equation (30) would predict a pressure of $245 \mathrm{~kg} / \mathrm{cm}^{2}$, assuming again that $\alpha$ is about 0.9 . The pressure that is developed when a flat solid surface strikes a cylindrical water surface should be between that which is developed when it strikes a flat water surface and that which results when it strikes a spherical water surface. De Haller's experimental measurement shows that this is essentially the case.

\section{Equation for the Flow Velocity at Maxi- mum Pressure}

Experimental measurements of the radius of flow of a waterdrop at regular intervals of time after collision at its terminal velocity in air with a glass surface were discussed in section 2.5. Figure 11 contains the curve of radial flow velocity against time. The data from which this curve was plotted are given in table 1 . With eq (30) of section 3 the radial flow velocity at the time of maximum pressure can be calculated.

Assume that the radial flow from an impinging waterdrop has axial symmetry and that the effect of viscosity may be neglected. Because the pressure at the periphery of the radial flow is atmospheric and may be taken as zero, and because the center of the flowing disk is a stagnation point, the equation of flow in cylindrical coordinates is

$$
P=\frac{\rho}{2} \int_{0}^{v_{0}} v d v+\rho \int_{0}^{r_{0}} \frac{\partial v}{\partial t} d r
$$

where $P$ is the pressure at the center of the disk, $\rho$ is the density of the liquid, $v_{0}$ is the observed velocity of spread, and $r_{0}$ is the observed radius of spread. It may be assumed that at the instant of maximum pressure $\partial v / \partial t$ is zero. Integration and substitution of the expression for $P$ given by eq (30) then yields

$$
v_{0}=[\alpha c V]^{1 / 2} .
$$

The value of $\alpha$ can be calculated from the velocity at which the glass surface moved through the waterdrop. This velocity is $(1-\alpha) V$. The central and side high lights on the waterdrop for the collision incident shown in figure 7 merged in frame 14, which was $0.0011_{8}$ sec from the point of impact or time zero. As the average waterdrop from the pipet that was used is $0.57 \mathrm{~cm}$ in diameter, the velocity at which the glass moved through the waterdrop was $484 \mathrm{~cm} / \mathrm{sec}$. The terminal velocity for a large waterdrop [4] is about $26.9 \mathrm{ft} / \mathrm{sec}(820 \mathrm{~cm} / \mathrm{sec})$. Hence,

$$
\begin{aligned}
(1-\alpha) 820 & =484, \\
\alpha & =0.4 .
\end{aligned}
$$

From eq (32), assuming that $c$ is about $5,000 \mathrm{ft} / \mathrm{sec}$, $V$ about $26.9 \mathrm{ft} / \mathrm{sec}$, and using the value of 0.4 for $\alpha$, the radial flow velocity for this waterdrop at the time of maximum pressure was $232 \mathrm{ft} / \mathrm{sec}$, or $8.6 \mathrm{~V}$. In order to plot this point on the velocity-againsttime curve shown in figure 11 it is necessary to know the time at which maximum pressure existed, that is, $\Delta t$ must also be evaluated. From eq (9),

$$
\delta=(1-\alpha) V \Delta t
$$

From eq (7) and (15)

$$
\delta=4 r(1-\alpha)^{2}[V / c]^{2} .
$$

From eq (7), (9), and (15)

$$
\Delta t=4 r(1-\alpha) V / c^{2} .
$$

The radius $r^{\prime}$ of the waterdrop was about $0.29 \mathrm{~cm}$, so that the radius of the flattened waterdrop from eq (11) was about $0.5 \mathrm{~cm}$. Using the value of 0.4 for $\alpha, \Delta t=0.04 \mu \mathrm{sec}$. The calculated velocity is plotted on the velocity-against-time curve shown in figure 11 and is enclosed in a circle to distinguish it from the measured values.

The radial flow of the waterdrop is a direct consequence of the impact force. This force goes to zero in about $1 \mathrm{msec}$ as is apparent from figure 10 . The head of water of the drop also goes to zero in approximately this same time interval, and the radial flow velocity drops from 8.6 times the value of the impact velocity, $V$, to less than $V$. After this, the water in radial flow continues to move outward under its own momentum until the flow is completely checked by its internal resistance and by surface tension.

\section{Equation for the Time Dependence of the Flow Radius}

The observed values of the radius of flow at regular intervals of time for the spread of a drop of water after collision at its terminal velocity in air against a glass plate are shown graphically in figure 11. It is possible to calculate the radius of flow.

At the instant at which it just collides with the glass plate, the energy of the falling waterdrop consists of its kinetic energy and its potential surface energy. As soon as it begins to spread on the glass, the kinetic energy begins to be transformed into potential energy of surface and into dissipated energy. As long as any part of the head of water remains, the 
terms of the energy equation are (1) the energy (kinetic and potential) remaining in the head of water, (2) the potential energy of surface of the radial flow, (3) the kinetic energy of the radially flowing water, and (4) the dissipated energy. At any instant of time the sum of these energy terms must be equal to the total energy of the falling drop at the instant of its collision with the glass plate.

The first of these energy terms would be difficult to evaluate. However, for all times after the head of water has vanished, that is, for all times during which the radial flow is moving outward under its own momentum, this energy term is zero. This is the case that will be considered.

At any instant at which an observation is made, the potential energy of surface of the radial flow, $P E$, is given by

$$
P E=\left(\gamma_{a}+\gamma_{g}\right) \pi r_{0}^{2}
$$

where $\gamma_{a}$ is the surface tension of water against air, $\gamma_{g}$ is the interfacial tension between water and glass, and $r_{0}$ is the observed value of the radius of spread.

To develop an expression for the kinetic energy it is necessary to make some assumption about the shape of the cross section of the radial flow. From the work of Worthington [1] it appears that the flow of a drop of milk is shallowest in the center and deepest at the periphery. Figure 15 (a) is an enlarged reproduction of one of the sketches given by Worthington. As a simple approximation to this shape the assumption has been made that the radial flow is an extremely flat cylinder except for an empty cone that extends through half the thickness of the cylinder. The assumed cross section of the flow is shown in figure $15(\mathrm{~b})$.

The kinetic energy, $K E$, at the instant when the radius is $r_{0}$ is given by

$$
K E=\frac{\rho}{2} \int_{0}^{r_{0}} 2 \pi r h d r \cdot v^{2}
$$

where $h$ and $v$ are the thickness of the flow and the radial flow velocity, respectively, and $\rho$ is the density of the water. As $v$ is zero at the center of the flow, and $v$ is $v_{0}$ at the periphery of the flow,

$$
v=\frac{r}{r_{0}} v_{0},
$$

where

$$
v_{0}=r_{0} / t \text {. }
$$

Thickness goes from $h_{0} / 2$ at the center of the flow to $h_{0}$ at the periphery, so that

$$
h=\frac{r}{r_{0}} \frac{h_{0}}{2}+\frac{h_{0}}{2}
$$

In terms of the mass, $M$, of the drop,

$$
h_{0}=6 M /\left[5 \pi \rho r_{0}^{2}\right],
$$

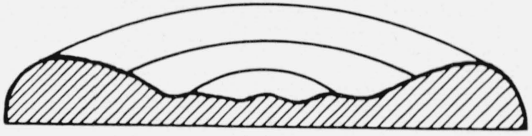

(a)

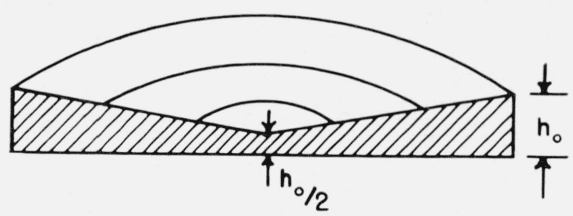

(b)

Figure 15. Cross section of the flow of a liquid drop.

(a) Cross section of the flow of a drop of milk as observed by Worthington; (b) assumed cross section of the flow of a drop of water.

since the volume of water is $\frac{5}{6}\left(\pi r_{0}^{2} h_{0}\right)$. Substituting the expressions for $v, h$, and $h_{0}$ in the expression for the kinetic energy,

$$
K E=\frac{54}{200} M \frac{r_{0}^{2}}{t^{2}} \sim \frac{M}{4} \frac{r_{0}^{2}}{t^{2}}
$$

At any instant of observation the dissipated energy, $D E$, is the accumulated dissipation during the time interval $t$. This dissipation is

$$
D E=2 \pi \int_{0}^{t} \int_{0}^{\Omega} \Phi d \Omega d t .
$$

where $\Phi$ is the rate of dissipation of energy per unit time per unit volume, and $\Omega$ is the volume of the boundary layer attached to the solid. The thickness of this layer is $\sigma$, and $\sigma$ is roughly less than $h_{0}$. Since in an axially symmetrical flow it suffices to consider only the variation of the radial flow velocity $v$ with the flow thickness $z$, for this case

$$
\Phi=\mu\left(\frac{\partial^{v}}{\partial^{z}}\right)^{2},
$$

where $\mu$ is the viscosity. Resorting to dimensional analysis and eq (41) and (42), it is seen that

$$
D E=C \int_{0}^{t}\left(\frac{v_{0}}{\sigma}\right)^{2} r_{0}^{2} \sigma d t
$$

where $r_{0}$ is the observed radius of flow and $C$ is a constant containing the viscosity $\mu$ and having the dimensions of viscosity. It may be supposed that $\sigma$ varies linearly with $t$, and, since $v_{0}$ is $r_{0} / t$,

$$
D E=k \int_{0}^{t} \frac{r_{0}^{4}}{t^{3}} d t
$$

where $k$ is a new constant.

The energy equation after the head of water remaining of the drop has vanished is

$$
\pi\left(\gamma_{a}+\gamma_{g}\right) r_{0}^{2}+\frac{M}{4} \frac{r_{0}^{2}}{t^{2}}+k \int_{0}^{t} \frac{r_{0}^{4}}{t^{3}} d t=\frac{M V^{2}}{2}+4 \pi r^{\prime 2} \gamma_{a}
$$


where $r^{\prime}$ is the radius of the original drop, and $V$ is the velocity at which the waterdrop strikes the surface. Differentiation with respect to time produces

$$
2 \pi\left(\gamma_{a}+\gamma_{g}\right) r_{0} \frac{d r_{0}}{d t}+\frac{M}{2} \frac{r_{0}}{t^{2}} \frac{d r_{0}}{d t}-\frac{M}{2} \frac{r_{0}^{2}}{t^{3}}+\frac{k r_{0}^{4}}{t^{3}}=0 .
$$

The solution of this equation is

$$
\frac{r_{0}^{2}}{\frac{M}{2}-k r_{0}^{2}}=\frac{t^{2}}{\frac{M}{2}+2 \pi\left(\gamma_{a}+\gamma_{g}\right) t^{2}} K,
$$

where $K$ is an integration constant. The expression for the integration constant is found from the condition that when $r_{0}=r_{\max }, t=t_{\max }$, where $r_{\max }$ indicates the maximum spread of the drop and $t_{\max }$ the corresponding time. Substituting the expression for the integration constant in eq (47), the equation for $r_{0}$ in terms of the time and constants is

$$
r_{0}^{2}=\frac{t^{2}\left(\frac{M}{2}-k r_{0}^{2}\right)}{\frac{M}{2}+2 \pi\left(\gamma_{a}+\gamma_{g}\right) t^{2}}\left[\frac{r_{\max }^{2}\left\{\frac{M}{2}+2 \pi\left(\gamma_{a}+\gamma_{g}\right) t_{\max }^{2}\right\}}{t_{\max }^{2}\left\{\frac{M}{2}--k r_{\max }^{2}\right\}}\right] .
$$

The equation is valid for all times after the head of water of the drop has vanished until the maximum spread is reached. It is necessary to evaluate the constant $k$ from the data. For the collision incident shown in figure 7 the central and side high lights on the waterdrop merge, that is, the head of water remaining of the drop has vanished at frame 14 . At frame $14, r_{0}=1.6_{1} \mathrm{~cm}, t=0.0011_{8}$ sec. The mass $M$ of an average drop from the pipet that was used is 0.0989 g. The maximum spread was reached at frame 44 , where $r_{0}=r_{\max }=2.3_{0} \mathrm{~cm}$ and $t=t_{\max }=$ $0.0040_{1}$ sec. At present there is no way of measuring directly the interfacial tension between water and glass experimentally, and the value of $2 \pi\left(\gamma_{a}+\gamma_{g}\right)$ can only be estimated roughly. Measurements of the surface tension of liquid glass against air have been made. Morey [7] lists the following values for a commercial soda-lime-silica glass: $404.3 \mathrm{~d} / \mathrm{cm}$ at $1,225^{\circ} \mathrm{C}, 406.2 \mathrm{~d} / \mathrm{cm}$ at $1,125^{\circ} \mathrm{C}$, and $407.8 \mathrm{~d} / \mathrm{cm}$ at $1,025^{\circ} \mathrm{C}$. From these data it appears that the value may be about $420 \mathrm{~d} / \mathrm{cm}$ at room temperature. It is impossible to know and hence to take into account any change that may be associated with the increased rigidity of the glass on cooling. Antonow's rule states [8] that for two liquids that are mutually saturated with each other, the interfacial tension is equal to the difference between the surface tensions of the two liquids separately. To this degree of approximation, $2 \pi\left(\gamma_{a}+\gamma_{g}\right) \sim 2,638 \mathrm{~d} / \mathrm{cm}$. This method of approximating the value of $\left(\gamma_{a}+\gamma_{g}\right)$ was suggested by William W. Walton of NBS Surface Chemistry Section.

To evaluate $k$, eq (48) was put in the form

$$
\frac{\frac{M}{2 r_{\max }^{2}}-k}{\frac{M}{2 r_{0}^{2}}-k}=\frac{\frac{M}{2 t_{\max }^{2}}+2 \pi\left(\gamma_{a}+\gamma_{g}\right)}{\frac{M}{2 t^{2}}+2 \pi\left(\gamma_{a}+\gamma_{g}\right)} .
$$

Substitution of the values of $M, r_{\max }, r_{0}$ at frame 14 , $t_{\max }, t$ at frame 14 , and $2 \pi\left(\gamma_{a}+\gamma_{g}\right)$ produced the result, $k=0.0076_{7} \mathrm{cgs}$ units.

The values of $r_{0}$ calculated by use of eq (48) are listed in table 1 with the measured values. The measured values have been multiplied by a magnifying factor. The method of determining this factor is discussed in section 2.5. The calculated values are in almost perfect agreement with the empirical values, which appears to justify, a posteriori, the assumptions that were made. The calculated values of $r_{0}$ are indicated with crosses in figure 11 .

In addition to specific acknowledgments that have been made in the text, the author thanks J. H. McMillan of the National Science Foundation, V. G. Szebehely of the David Taylor Model Basin, and George Irwin of the Naval Research Laboratory for conversations in which valuable suggestions were made.

The work described in this paper was sponsored by the Wright Air Development Center and was taken from reports submitted to them. Their assistance and interest are gratefully acknowledged.

\section{References}

[1] A. M. Worthington, On the forms assumed by drops of liquids falling vertically on a horizontal plate, Proc. Roy. Soc. 25, 261 (1877); 25, 498 (1877).

[2] A. M. Worthington, A study of splashes (Longmans, Green, and Co., New York, N. Y., 1908).

[3] R. T. Knapp and A. Hollander, Laboratory investigations of the mechanism of cavitation, Trans. ASME $\mathbf{y}_{0}$, 419 (1948).

[4] D. C. Blanchard, General Electric Research Laboratory Occasional Report No. 7, Project Cirrus (November 1948).

[5] E. Honegger, Tests on erosion caused by jets, Brown Boveri Rev. 14. 95 (1927).

[6] P. de Haller, Untersuchungen über die durch Kavitation hervorgerufenen Korrosionen, Schweiz. Bauztg. 101, 243 (1933) ; 101, 260 (1933).

7] George W. Morey, Properties of glass, p. 201 (Reinhold Publishing Corp., New York, N. Y., 1945).

[8] N. K. Adam, Physics and chemistry of surfaces, p. 215 (Oxford University Press, London, 1941).

Washington, September 30, 1954. 